\title{
APASS LANDOLT-SLOAN BVgri PHOTOMETRY OF RAVE STARS. I. DATA, EFFECTIVE TEMPERATURES, AND REDDENINGS
}

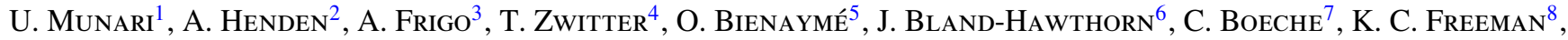 \\ B. K. Gibson ${ }^{9,10}$, G. Gilmore ${ }^{11}$, E. K. Grebel ${ }^{7}$, A. Helmi ${ }^{12}$, G. Kordopatis ${ }^{11}$, S. E. Levine ${ }^{13}$, J. F. Navarro ${ }^{14}$, \\ Q. A. Parker ${ }^{15}, 16$, W. Reid ${ }^{15,17}$, G. M. Seabroke ${ }^{18}$, A. Siebert ${ }^{5}$, A. Siviero ${ }^{1}$, T. C. Smith ${ }^{19}$, M. Steinmetz $^{20}$, \\ M. Templeton ${ }^{2}$, D. Terrell ${ }^{21}$, D. L. Welch ${ }^{22}$, M. Williams ${ }^{20}$, And R. F. G. Wyse ${ }^{23}$ \\ ${ }^{1}$ INAF Osservatorio Astronomico di Padova, I-36012 Asiago (VI), Italy \\ ${ }^{2}$ AAVSO, Cambridge, MA, USA \\ ${ }^{3}$ ANS Collaboration, c/o Astronomical Observatory, Padova, Italy \\ ${ }^{4}$ Faculty of Mathematics and Physics, University of Ljubljana, 1000 Ljubljana, Slovenia \\ ${ }^{5}$ Observatoire Astronomique, Université de Strasbourg, CNRS, 11 rue de l'université F-67000 Strasbourg, France \\ ${ }^{6}$ Sydney Institute for Astronomy, University of Sydney, NSW 2006, Australia \\ ${ }^{7}$ Astronomisches Rechen-Institut, Zentrum für Astronomie der Universität Heidelberg, Mönchhofstr. 12-14, D-69120 Heidelberg, Germany \\ ${ }^{8}$ Mount Stromlo Observatory, RSAA, Australian National University, Weston Creek, Canberra, ACT 2611, Australia \\ ${ }^{9}$ Jeremiah Horrocks Institute, University of Central Lancashire, Preston, PR1 2HE, UK \\ ${ }^{10}$ Institute for Computational Astrophysics, Dept of Astronomy and Physics, Saint Marys University, Halifax, NS, BH3 3C3, Canada \\ ${ }^{11}$ Institute of Astronomy, Cambridge University, Madingley Road, Cambridge CB3 OHA, UK \\ 12 Kapteyn Astronomical Institute, University of Groningen, PO Box 800, 9700 AV Groningen, The Netherlands \\ ${ }^{13}$ Lowell Observatory, Flagstaff, AZ, USA \\ ${ }^{14}$ Department of Physics and Astronomy, University of Victoria, Victoria, BC V8P 5C2, Canada \\ ${ }_{15}^{15}$ Department of Physics \& Astronomy, Macquarie University, NSW 2109, Australia \\ ${ }^{16}$ Australian Astronomical Observatory, PO Box 915, North Ryde, NSW 1670, Australia \\ ${ }_{17}$ Macquarie Research Centre in Astronomy, Astrophysics and Astrophotonics, Macquarie University, NSW 2109, Australia \\ ${ }_{18}$ Mullard Space Science Laboratory, University College London, Holmbury St. Mary, Dorking, RH5 6NT, UK \\ ${ }^{19}$ Dark Ridge Observatory, Weed, New Mexico, NM, USA \\ ${ }^{20}$ Leibniz-Institut für Astrophysik Potsdam (AIP), An der Sternwarte 16, D-14482 Potsdam, Germany \\ ${ }_{21}$ Southwest Research Institute, Boulder, CO, USA \\ ${ }^{22}$ McMaster University, Hamilton, Ontario, Canada \\ ${ }^{23}$ Johns Hopkins University, Homewood Campus, 3400 North Charles Street, Baltimore, MD 21218, USA \\ Received 2014 May 29; accepted 2014 July 25; published 2014 September 26
}

\begin{abstract}
We provide AAVSO Photometric All-Sky Survey (APASS) photometry in the Landolt $B V$ and Sloan $g^{\prime} r^{\prime} i^{\prime}$ bands for all 425,743 stars included in the fourth RAVE Data Release. The internal accuracy of the APASS photometry of RAVE stars, expressed as the error of the mean of data obtained and separately calibrated over a median of four distinct observing epochs and distributed between 2009 and 2013, is $0.013,0.012,0.012,0.014$, and 0.021 mag for the $B, V, g^{\prime}, r^{\prime}$, and $i^{\prime}$ bands, respectively. The equally high external accuracy of APASS photometry has been verified on secondary Landolt and Sloan photometric standard stars not involved in the APASS calibration process and on a large body of literature data on field and cluster stars, confirming the absence of offsets and trends. Compared with the Carlsberg Meridian Catalog (CMC-15), APASS astrometry of RAVE stars is accurate to a median value of 0.098 arcsec. Brightness distribution functions for the RAVE stars have been derived in all bands. APASS photometry of RAVE stars, augmented by 2MASS $J H K$ infrared data, has been $\chi^{2}$ fitted to a densely populated synthetic photometric library designed to widely explore temperature, surface gravity, metallicity, and reddening. Resulting $T_{\text {eff }}$ and $E_{B-V}$, computed over a range of options, are provided and discussed, and will be kept updated in response to future APASS and RAVE data releases. In the process, we find that the reddening caused by a homogeneous slab of dust, extending for $140 \mathrm{pc}$ on either side of the Galactic plane and responsible for $E_{B-V}^{\text {poles }}=0.036 \pm 0.002$ at the Galactic poles, is a suitable approximation of the actual reddening encountered at Galactic latitudes $|b| \geqslant 25^{\circ}$.
\end{abstract}

Key words: catalogs - methods: data analysis - surveys - techniques: photometric

Online-only material: color figures, machine-readable and VO tables

\section{INTRODUCTION}

The RAdial Velocity Experiment (RAVE) is a digital spectroscopic survey of stars in the nominal magnitude interval $9 \leqslant I_{\mathrm{C}} \leqslant 12$, distributed over the whole southern sky. Spectra have been recorded over the 8400-8800 $\AA$ range, at an average resolving power $\sim 6500$, with the Australian Astronomical Observatory (AAO) UK Schmidt telescope feeding light to a spectrograph via the six-degree field $(6 \mathrm{dF}) 150$ fiber positioner.
The primary science driver of the survey is the investigation of the structure and evolution of the Milky Way through the determination of radial velocities, chemistries, temperatures, and gravities for a large number of stars. The survey data releases (Steinmetz et al. 2006; Zwitter et al. 2008; Siebert et al. 2011) have followed an incremental scheme, with the last Data Release N.4 (DR4 Kordopatis et al. 2013) covering 482,194 spectra for 425,743 individual stars. RAVE data have so far directly supported about 50 refereed papers. 
Accurate photometry, based on bands spanning the optical and near-infrared wavelength ranges, is an essential support for spectroscopic surveys like RAVE. Such photometry provides, among other things: (1) the brightness distribution functions of the survey targets, (2) the effective temperature, useful during or prior to the spectroscopic determination of atmospheric parameters, (3) the reddening, (4) the observed brightness used to derive the distance modulus $(m-M)$ to the survey targets, (5) the color discriminant that segregates single normal stars from binaries and peculiar stars, and (6) if multi-epoch photometry is available, information on the target intrinsic variability. So far, only limited photometric information in the optical has been available for RAVE stars, as reviewed below. The goal of this paper is to provide accurate multi-band optical photometry for all RAVE stars and to combine it with near-IR 2MASS photometry.

A total of 251,626 RAVE DR4 stars have a match in the Tycho- $2 B_{T}, V_{T}$ catalog. Unfortunately, the Tycho- 2 photometric errors rapidly degrade toward the faint survey limit and over the range of brightness of the bulk of RAVE stars, Tycho-2 data (especially those in the $B_{T}$ band) are virtually useless. The median value of quoted Tycho-2 errors for RAVE stars is $\sigma\left(B_{T}\right)=0.15$ and $\sigma\left(V_{T}\right)=0.09 \mathrm{mag}$, for a combined $\sigma(B-V)_{T} \sim 0.18$ which is far larger than the reddening affecting the majority of RAVE stars or larger than the color spread corresponding to the combined uncertainty in the RAVE atmospheric parameters $\left(T_{\text {eff }}, \log g,[\mathrm{M} / \mathrm{H}]\right)$.

A greater number of RAVE DR4 stars, 407,061 in total, have a counterpart in the DENIS $I, J, K$ catalog. DENIS $J$ and $K$ photometry is of limited precision, the median value on RAVE stars being $\sigma(J)=0.07$ and $\sigma(K)=0.07 \mathrm{mag}$ (three times worse than corresponding 2MASS values), with $\sigma(J-K)=0.10$ on the combined color index $(\sigma(J-K)=0.033$ for $2 \mathrm{MASS})$. Such an uncertainty on the color index corresponds to an uncertainty of $15 \%$ in the effective temperature of a red giant star, or $0.20 \mathrm{mag}$ in $E_{B-V}$, spoiling the DENIS $J, K$ photometry of any practical value in the supporting RAVE survey. DENIS $I$ photometry, even if reported to a greater precision (a median of $\sigma(I)=0.03$ mag on RAVE stars), is affected by saturation problems for bright RAVE targets, setting in at undocumented different levels of brightness depending on the location on the sky (cf. Kordopatis et al. 2013). Furthermore, the transmission profile of the DENIS I band is not accurately known, thus the observed complex offset from Landolt and Cousins standard stars cannot be safely compensated for during modeling and analysis of the data.

A further source of photometry for a consistent fraction of RAVE stars is the $r^{\prime}$ band data obtained as a by-product during the astrometric operation of the Carlsberg Meridian Circle in La Palma, covering the sky between declinations $-40 \leqslant \delta \leqslant+50$. The final Carlsberg Meridian Circle data release, CMC-15 of 2013 , contains counterparts to 228,805 RAVE DR4 stars. The original CMC observations did not include a second photometric band, which is necessary for transformation from the local to the standard system, which was achieved by importing the bluer color $\left(B_{T}-V_{T}\right)$ from the Tycho- 2 catalog into the process. The median value of the CMC-15 reported internal error on $r^{\prime}$ band data for RAVE DR4 stars is $0.026 \mathrm{mag}$. The CMC-15 $r^{\prime}$ band data will be further considered in Section 2.5.

On the near-IR side, 2MASS $J, H$, and $K$ magnitudes are available for all RAVE DR4 stars. The median value of the quoted errors for RAVE stars is $\sigma(J)=0.024, \sigma(H)=$ $0.025, \sigma(K)=0.023 \mathrm{mag}$, much better than for the corre- sponding DENIS bands. However, even if 2MASS $J, H, K$ data are available for all RAVE stars, and they are of reasonable accuracy, nevertheless they map the Rayleigh-Jeans tail of the energy distributions of typical RAVE stars (cf. Figure 1), which provides only a limited leverage in terms of diagnostic capability. In fact, the 10-90 percentile interval in the color distribution of RAVE stars corresponds to just $\Delta(J-K)=0.87 \mathrm{mag}$.

The aim of the present paper is to provide accurate photometry for all RAVE DR4 stars in the Landolt $B, V$ and Sloan $g^{\prime}, r^{\prime}, i^{\prime}$ photometric bands, as obtained during the AAVSO Photometric All-Sky Survey(APASS; Henden \& Munari 2014; Henden et al. 2014). Combined with 2MASS $J, H, K$ data in the infrared, the APASS photometric bands encompass the majority of the energy distribution of typical RAVE stars, as shown schematically in Figure 1. The plan of this paper is first to present the APASS data on RAVE stars and test their photometric and astrometric quality. Photometric degeneracy between temperature and reddening is then discussed, and a simple model for galactic extinction is introduced and its parameters are calibrated against APASS+2MASS data for standard stars. Photometric temperatures are derived, first for a set of standard stars to assess their accuracy, and then for the whole body of RAVE stars. These photometric temperatures are compared with those derived spectroscopically, and the results are discussed. Finally, the $E_{B-V}$ reddening is derived for all RAVE DR4 stars both from an unconstrained fit as well as from fixing the atmospheric parameters to the values derived by RAVE spectra.

Among the goals of future papers in this series will be the derivation of a Galactic 3D map for the interstellar $E_{B-V}$ reddening derived for RAVE stars, the photometric characterization of RAVE peculiar stars, the study of the photometric variability of RAVE peculiar stars by accessing the individual epoch photometric data, the use of supplementary observations in the $u^{\prime}, z^{\prime}$, and $Y$ filters, and extension to magnitudes brighter than the current APASS saturation limits.

\section{THE DATA}

\subsection{The APASS Survey}

The $B V g^{\prime} r^{\prime} i^{\prime}$ photometric data of RAVE stars discussed in this paper have been obtained as part of the ongoing APASS survey (Henden \& Munari 2014; Henden et al. 2014). The all-sky APASS survey is carried out to fill the gap existing between the completeness limit of the all-sky Tycho-2 $B_{T} V_{T}$ survey $\left(V_{T} \sim 11\right)$ and the saturation limit $(V \sim 15)$ of planned or currently operating near-IR and optical photometric surveys such as SDSS, PanSTARRS, LSST, SkyMapper, VISTA, and VST. APASS results are already used as the photometric source catalog for the US Naval Observatory CCD Astrograph Catalog (UCAC-4), and for the Harvard College DASCH program (which is scanning the Harvard plate stacks and measuring the magnitude of all detected stars).

The APASS photometric survey covers the whole sky, from North Pole to South Pole, with ongoing observations from CTIO (Chile), for the southern hemisphere, and New Mexico for the northern counterpart. At both sites, a pair of twin, remotely controlled, small telescopes obtain simultaneous CCD observations during dark- and gray-Moon time over five optical bands: $B, V$ (tied to the equatorial standards of Landolt 2009) and $g^{\prime}, r^{\prime}, i^{\prime}$ (tied to the 158 primary standards given by Smith et al. (2002), which define the Sloan photometric system). 


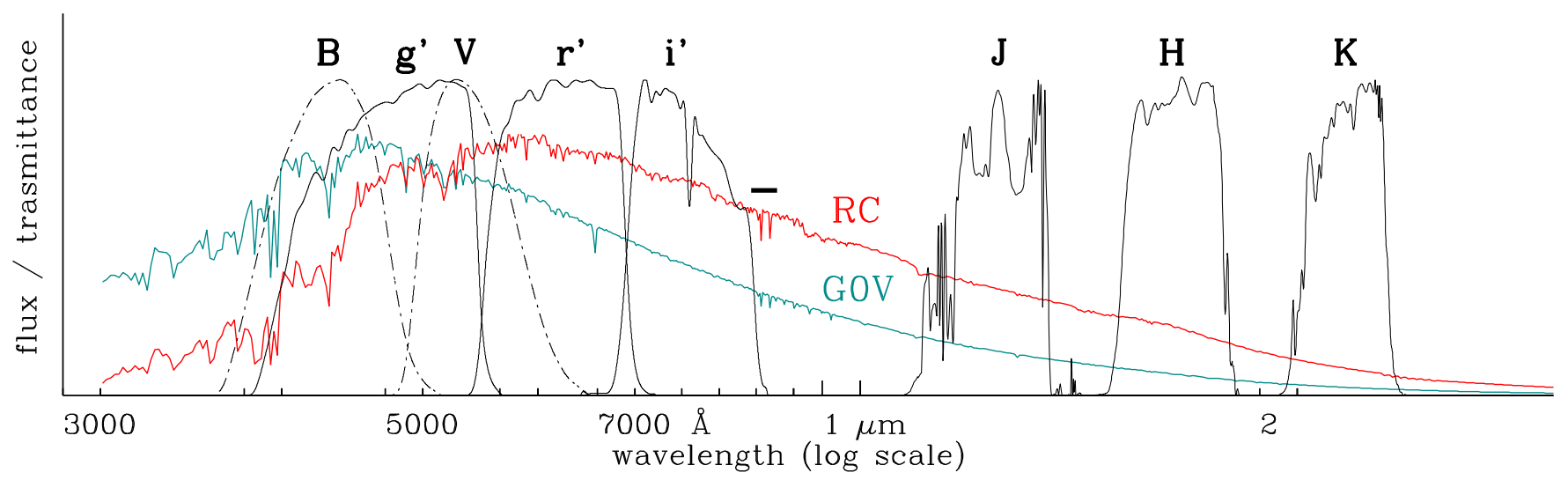

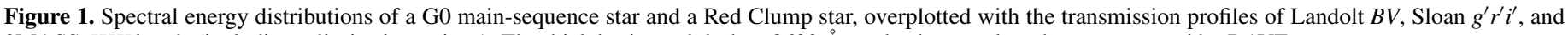
2MASS JHK bands (including telluric absorptions). The thick horizontal dash at $8600 \AA$ marks the wavelength range covered by RAVE spectra.

(A color version of this figure is available in the online journal.)

The telescopes are $20 \mathrm{~cm} f / 3.6$ astrographs feeding Apogee U16m cameras ( $4096 \times 4096$ array, $9 \mu \mathrm{m}$ pixels), that cover a field $2.9 \mathrm{deg}$ wide with a $2.6 \mathrm{arcsec}_{\text {pixel }}{ }^{-1}$ plate factor. The photometric filters are of the dielectric multi-layer type and are produced by Astrodon. Transmission curves and photometric performances of Astrodon filters are discussed and compared to more conventional types of photometric filters in Munari et al. (2012) and Munari \& Moretti (2012). On average, 80 fields are observed per night at each APASS location, 20 of them being standard fields (Landolt, Sloan) well distributed in time and air mass.

The APASS observations are obtained with fixed exposure times (different and optimized for each photometric band) set to detect $V=17$ stars at $\mathrm{S} / \mathrm{N}=5$ on a single exposure. Stars brighter than $V=10$ may saturate under optimal seeing conditions. APASS has also recently begun using the brightMoon periods to obtain shorter exposure observations in order to push the saturation limit upward to $7.5 \mathrm{mag}$ stars and also to observe in the $Y$ band at $1.035 \mu \mathrm{m}$ (defined by Hillenbrand et al. 2002; Sharon et al. 2010) and the Sloan $u^{\prime}, z^{\prime}$ bands.

\subsection{Astrometric Matching}

The astrometric matching to the APASS catalog has been performed by taking the positions and proper motions of RAVE stars as listed in DR4 and projecting them to the 2013 APASS epoch. The matching radius has been set to 3.0 arcsec. All 425,743 RAVE DR4 stars turned out to have APASS counterparts.

As a test on the accuracy of APASS astrometry (which is calibrated against UCAC-4), we have searched the final CMC-15 release (2013) of the Carlsberg Meridian Catalog for the positions of RAVE stars and computed the separation between the APASS and Carlsberg positions (equinox J2000, individual epochs varying from 1999 to 2005). Their distribution is presented in Figure 2. The peak of this distribution is at a separation of $0.072 \mathrm{arcsec}$ and the median is at $0.098 \mathrm{arcsec}$, confirming the high astrometric accuracy of APASS products.

\subsection{Internal Photometric Accuracy}

Each APASS observing night is independently calibrated in all-sky mode against the Landolt and Sloan standards observed that same night over a large range of air mass. The photometric quality of a given night can be evaluated only a posteriori during the data reduction process, and only data obtained during

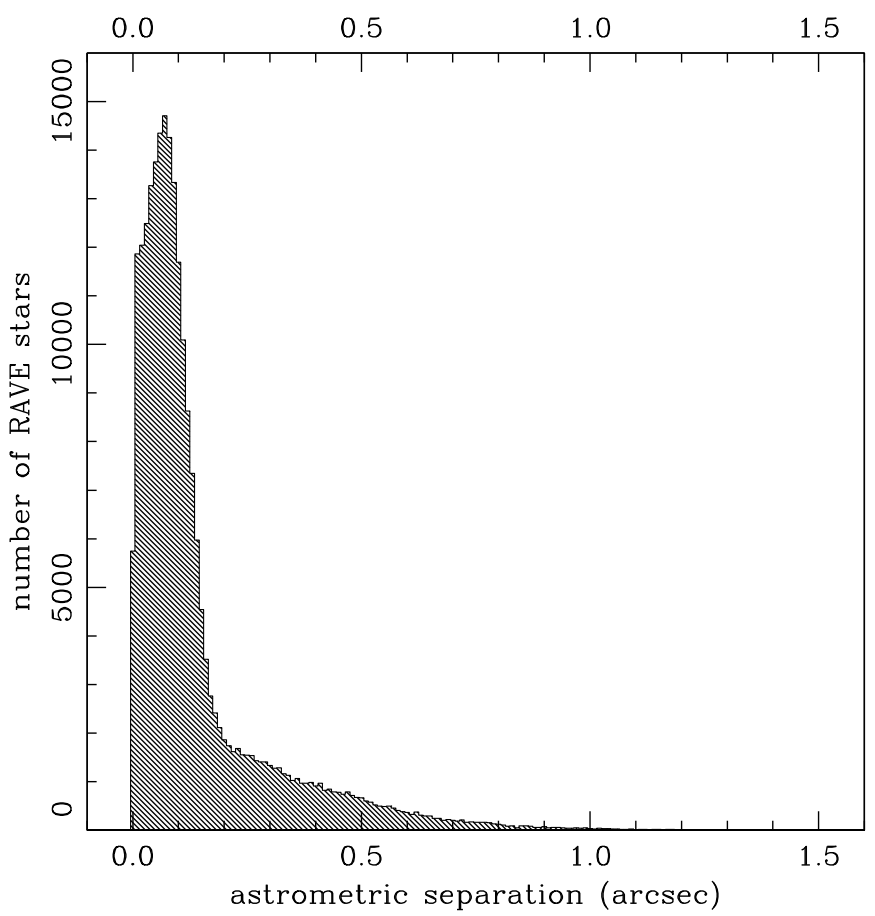

Figure 2. Distribution of the differences (in arcseconds) between the astrometric positions derived by APASS for the RAVE stars and the positions listed for the same stars in the latest issue (CMC-15 from 2013) of the Carlsberg Meridian Catalog.

photometric nights are used to build the APASS catalog. The difference in magnitude for data on the same stars obtained in separate nights (usually months apart), is therefore a reliable estimate of the total internal errors affecting APASS photometry. Their distribution for RAVE stars is presented in Figure 3, and the distribution of RAVE stars as a function of the number of epochs of APASS observations is given in Figure 4 (on average, a RAVE star has been observed in four independent epochs by APASS and none at less than two epochs). The errors corresponding to the tails of the distributions in Figure 3 are actually an overestimate of the true internal photometric errors. In fact, in building Figure 3, all stars are treated as nonvariable, but as shown by the Hipparcos satellite, about $10 \%$ of all stars it observed actually showed a variability of at least $0.01 \mathrm{mag}$ amplitude. Median values of the error distributions in Figure 3, 


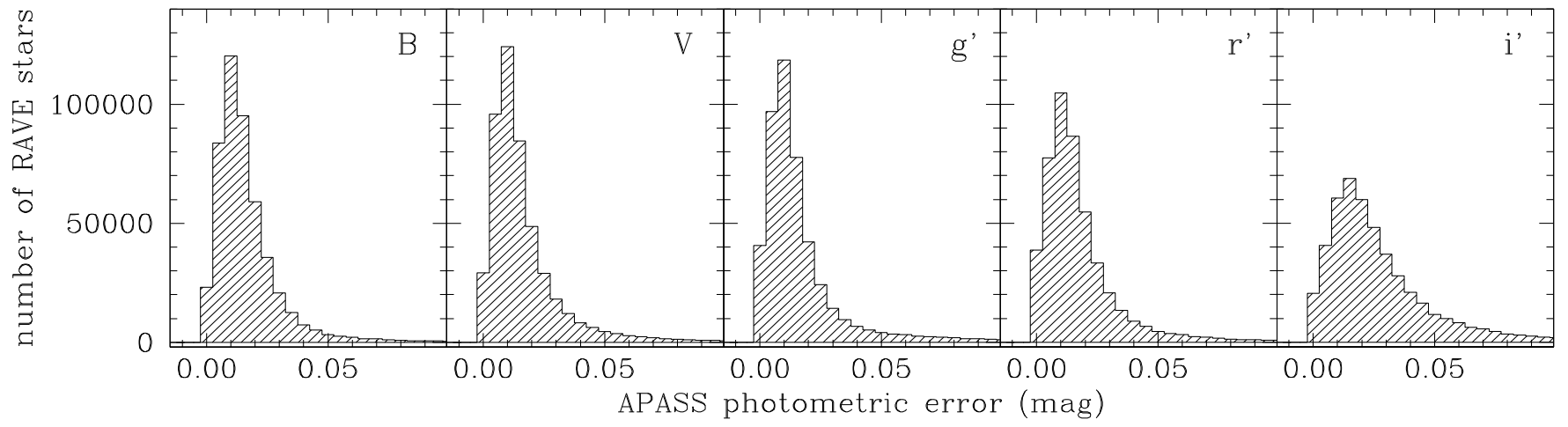

Figure 3. Distribution of errors of APASS $B V g^{\prime} r^{\prime} i^{\prime}$ magnitudes for RAVE stars (errors of the mean).

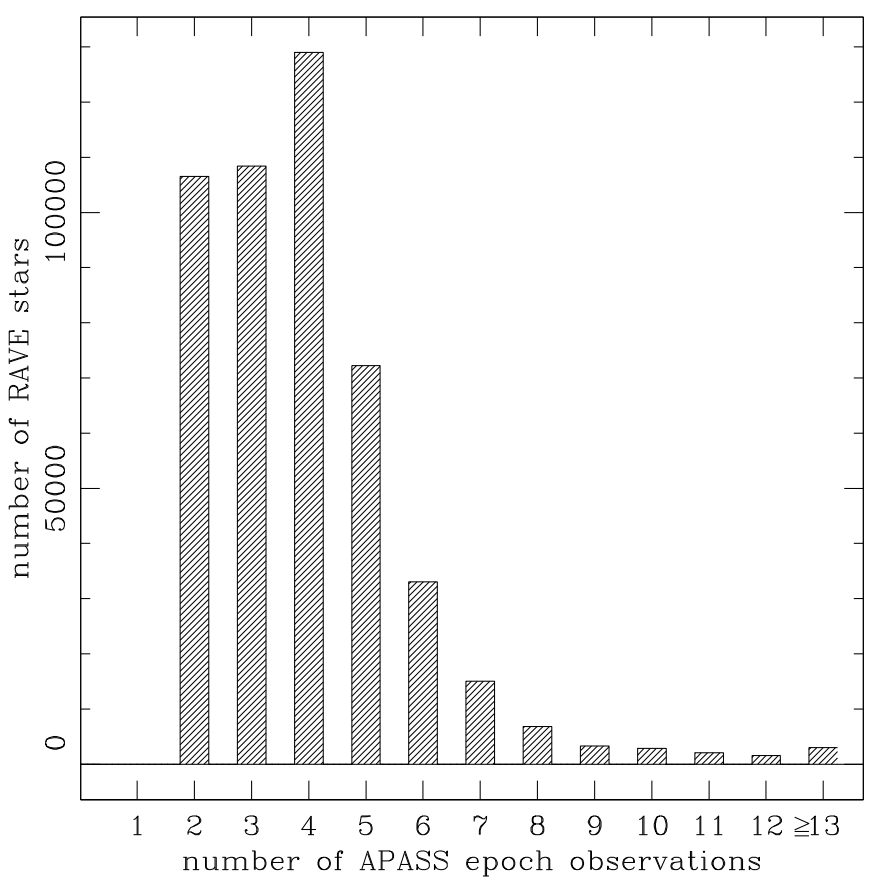

Figure 4. Number of independent epochs in APASS observations of RAVE stars.

and the corresponding 2MASS values are:

$$
\begin{aligned}
& \operatorname{err}(B)=0.013 \mathrm{mag} \\
& \operatorname{err}(V)=0.012 \mathrm{mag} \\
& \operatorname{err}\left(g^{\prime}\right)=0.012 \mathrm{mag} \\
& \operatorname{err}\left(r^{\prime}\right)=0.014 \mathrm{mag} \\
& \operatorname{err}\left(i^{\prime}\right)=0.021 \mathrm{mag} \\
& \operatorname{err}(J)=0.024 \mathrm{mag} \\
& \operatorname{err}(H)=0.025 \mathrm{mag} \\
& \operatorname{err}(K)=0.023 \mathrm{mag}
\end{aligned}
$$

where the larger value for the $i^{\prime}$ band (compared to $B, V, g^{\prime}$ and $r^{\prime}$ bands) is mainly due to the variable intensity of the strong telluric absorptions present over its pass-band. By the time the final products of the APASS survey will be released and the number of observing epochs doubled, the above errors are expected to reduce by $\sim 30 \%$.

\subsection{Saturation Limits}

A necessary test is to evaluate at what magnitude saturation sets in (because of the fixed exposure time over the whole
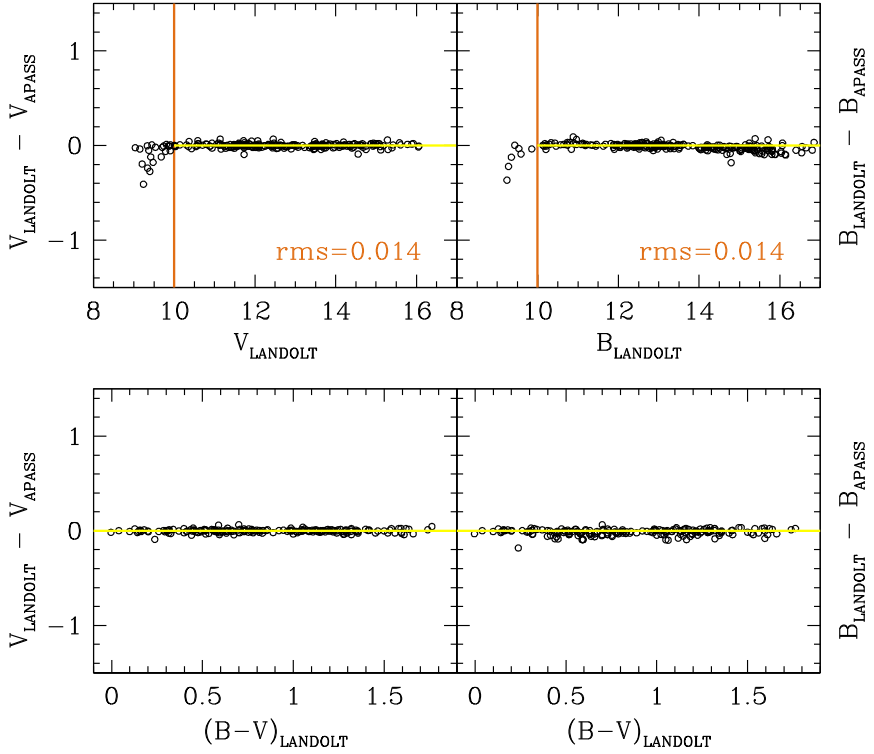

Figure 5. Comparison between the source $B, V$ data of the Landolt (2009) equatorial standards and the corresponding APASS values. The vertical line marks where saturation of brighter stars sets in (because of APASS fixed exposure times) under excellent seeing conditions. Saturated stars from top panels are not plotted on lower panels.

(A color version of this figure is available in the online journal.)

sky), and therefore to place a safe upper brightness limit on the use of APASS data to model properties of RAVE stars. The test is carried out in Figure 5 where the tabular value of 245 Landolt primary standards is compared to that derived by APASS (which obviously uses only nonsaturated Landolt standards to perform its calibration). Note that APASS uses the same 14 arcsec diameter digital aperture that matched the Landolt 14 arcsec photoelectric photometer aperture, so any neighboring star included in Landolt's aperture is also included in the APASS photometry. Figure 5 shows that most of the $B, V$ observations are unsaturated up to $9.5 \mathrm{mag}$, but excellent seeing can cause this limit to shift down to 10.0 mag. A similar exercise for Sloan 158 primary standards shows that the $i^{\prime}$ band is unsaturated up to $9.0 \mathrm{mag}$ and the $\mathrm{r}^{\prime}$ band up to $9.5 \mathrm{mag}$, while the limit for the $g^{\prime}$ band is $10.0 \mathrm{mag}$. These results perfectly match those independently derived from a different and independent set of APASS data during the search for and the characterization of RR Lyr variables potentially associated with the Aquarius stream (Munari et al. 2014), a Galactic stream originating from a disrupting globular cluster that was first identified on RAVE data (Williams et al. 2011; Wylie de Boer et al. 2012). 

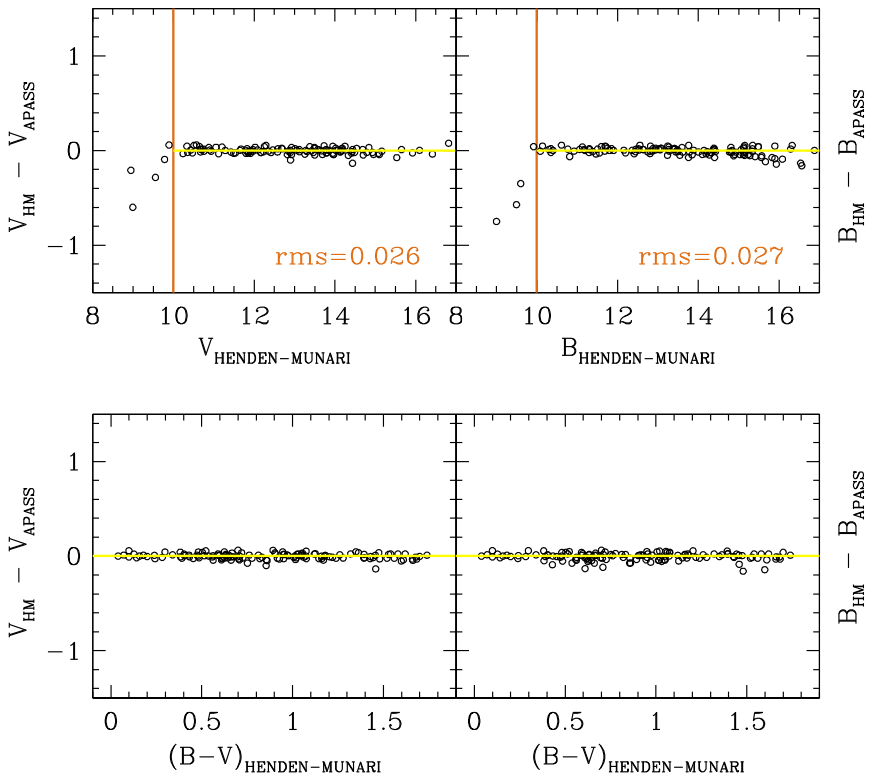

Figure 6. Comparison between the $B, V$ data of 323 secondary, low Galactic latitude Landolt standards calibrated by Henden \& Munari (2000, 2001, 2006) and the corresponding APASS values. Saturated stars from the top panels are not plotted on the lower panels.

(A color version of this figure is available in the online journal.)

To have a safe margin in all bands and for all stars, we will limit the analysis in the rest of this paper to stars with an APASS magnitude $\geqslant 10.0$ in the $B, V, g^{\prime}, r^{\prime}$ bands and $\geqslant 9.5$ in $i^{\prime}$ (only $1.8 \%$ of the total number of RAVE DR4 stars is consequently ignored in the following). As mentioned in the Introduction, APASS is currently using the bright-Moon nights to perform a shallower parallel survey aiming to push the saturation limit up to 7.5 mag stars. The APASS data for the brighter RAVE stars will be investigated later in this series of papers.

\subsection{External Photometric Accuracy}

Even if the small internal errors already make APASS the most accurate all-sky, multi-band photometric survey in the optical wavelength range, what really matters are the external errors. To estimate them, we compare APASS data to the available literature data. We begin with photometric standard stars not used in the calibration of APASS data (to avoid circular arguments), and then move to considering field and cluster stars.

Henden \& Munari (2000, 2001, 2006) have calibrated a series of secondary Landolt standards in 81 low Galactic latitude fields. These secondary standards are not used to calibrate APASS data, which rely only on the primary Landolt equatorial standards. A comparison with Henden \& Munari secondary standards is carried out in Figure 6. It shows no offsets or trends with APASS $B, V$ photometry, and confirms 10.0 mag as the saturation limit for the survey. The rms of APASS data on these secondary standards is somewhat larger than on the Landolt primary standards used for the photometric calibration. A reason for this is the large crowding affecting the low Galactic fields where the Henden \& Munari secondary standards are located, while Landolt standards are primarily high Galactic latitude stars well isolated from neighbors.

In addition to the primary 158 photometric standards for the Sloan system, Smith et al. (2002) also published a list of an additional 2988 secondary standards. These Sloan secondary standards are not used in the APASS calibration, so they
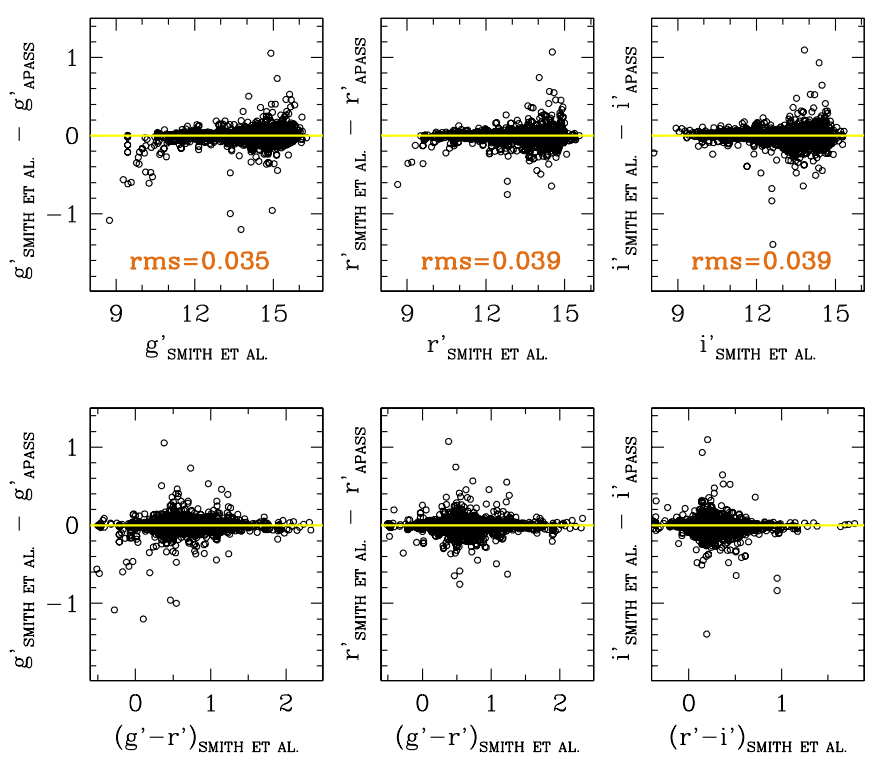

Figure 7. Comparison between the $g^{\prime} r^{\prime} i^{\prime}$ data of 2988 secondary Sloan standards provided by Smith et al. (2002) and the corresponding APASS values.

(A color version of this figure is available in the online journal.)

constitute an unbiased external consistency check for APASS photometry. A comparison between the two is carried out in Figure 7, and again shows no offset and no trend. The larger scatter is most likely due to the larger 24 arcsec digital aperture used by Smith et al. Their 158 primary standards were also mostly Landolt standards with no nearby neighbors, but the 2988 secondary standards may not have been so carefully selected.

While there are no large sets of stars in the RAVE brightness range that were measured by different authors in the $g^{\prime} r^{\prime} i^{\prime}$ Sloan bands, the Johnson $B, V$ bands have extensive coverage. A relevant source of data is the Catalog of Mean UBV Data (Cat$\mathrm{UBV}$, for short), critically assembled from existing literature by Mermilliod \& Mermilliod (1994) during the work leading to the compilation of the Hipparcos Input Catalog. It covers 103,108 stars with $U B V$ measurements spread over 693 different source papers $(27,803$ stars rely on only one observation from only one source). Figure 8 presents a comparison of the 723 field stars fainter than 10.0 mag in both $B$ and $V$ that are in common to both the Cat-UBV and APASS, and for which Cat-UBV averages over at least two distinct data sources. Figure 9 presents a similar comparison for 1073 stars that are members of clusters. What is relevant in these two figures is the absence of offsets and trends in both the magnitude zero points and their color dependences. The outliers in both figures are almost entirely stars with only one observation from just two independent sources. The rms values in Figures 8 and 9 are somewhat larger than for the comparisons carried out in Figures 5-7. There are various possible causes for this, such as (1) the presence of unrecognized low-amplitude variable stars, (2) the mainly photoelectric origin of Cat-UBV data (in photoelectric photometry all the light entering the generously large aperture of the photometer-usually tens of arcseconds - is measured, thus including the target together with faint field stars, while in CCD photometry the stars are isolated from the background and measured individually; perhaps not incidentally, data for members of open cluster appear more dispersed in Figure 9 than data for field stars in Figure 8), and (3) the many different variants over which independent authors whose data were entered into Cat-UBV tried to replicate the 

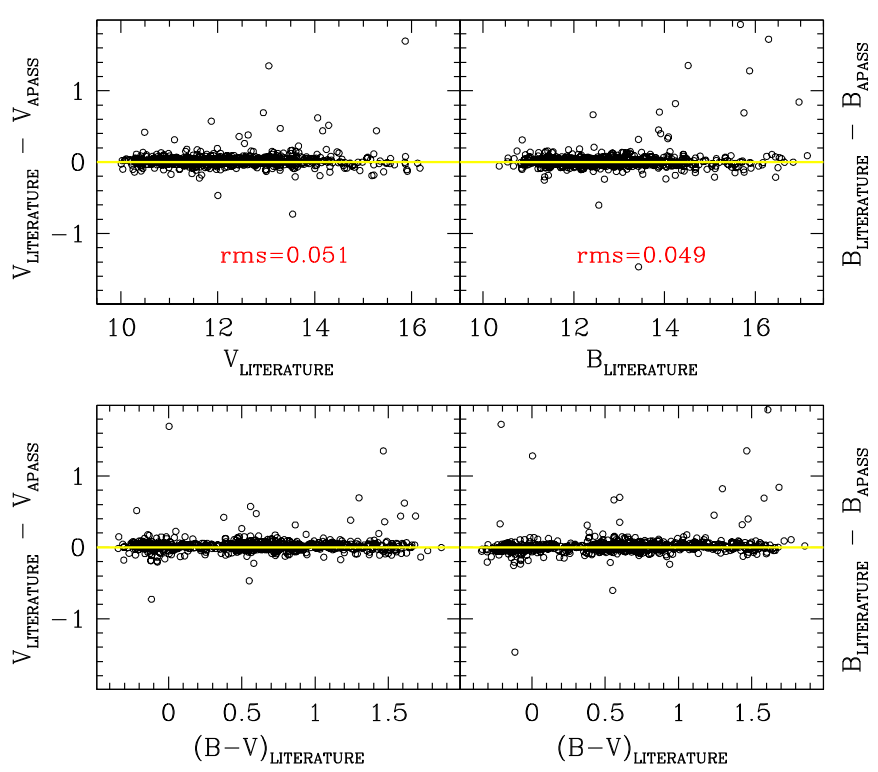

Figure 8. Comparison between literature $B, V$ data on field stars compiled by Mermilliod \& Mermilliod (1994) and the corresponding APASS values.

(A color version of this figure is available in the online journal.)
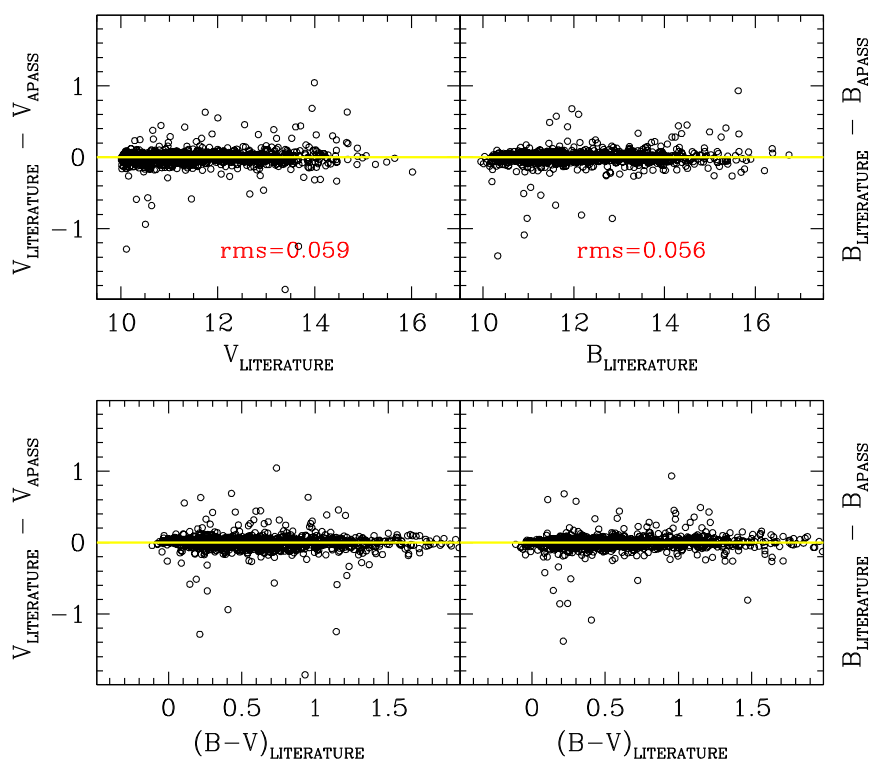

Figure 9. Comparison between literature $B, V$ data on stars belonging to open clusters as compiled by Mermilliod \& Mermilliod (1994), and the corresponding APASS values.

(A color version of this figure is available in the online journal.)

Johnson $B V$ bands, Landolt $B V$ bands being just one of these variants (a comprehensive discussion of the realization of the Johnson's system and the difficulties of reproducing it with instrumentation that changed with time, is given in Bessell 1990, in Golay (1974) and Straižys (1995), and the two volumes of the Asiago Database on Photometric Systems by Moro \& Munari (2000) and Fiorucci \& Munari (2003).

The comparison between the CMC-15 and APASS $r^{\prime}$ magnitudes for the 228805 RAVE DR4 stars common to both is presented in Figure 10. There is no trend with color, a minor systematic offset of just 0.009 mag, and a tight concentration of points with the quartile of the distribution amounting to only $0.018 \mathrm{mag}$. Considering the uncertainties of the photometry included in CMC-15 mentioned above, this comparison between

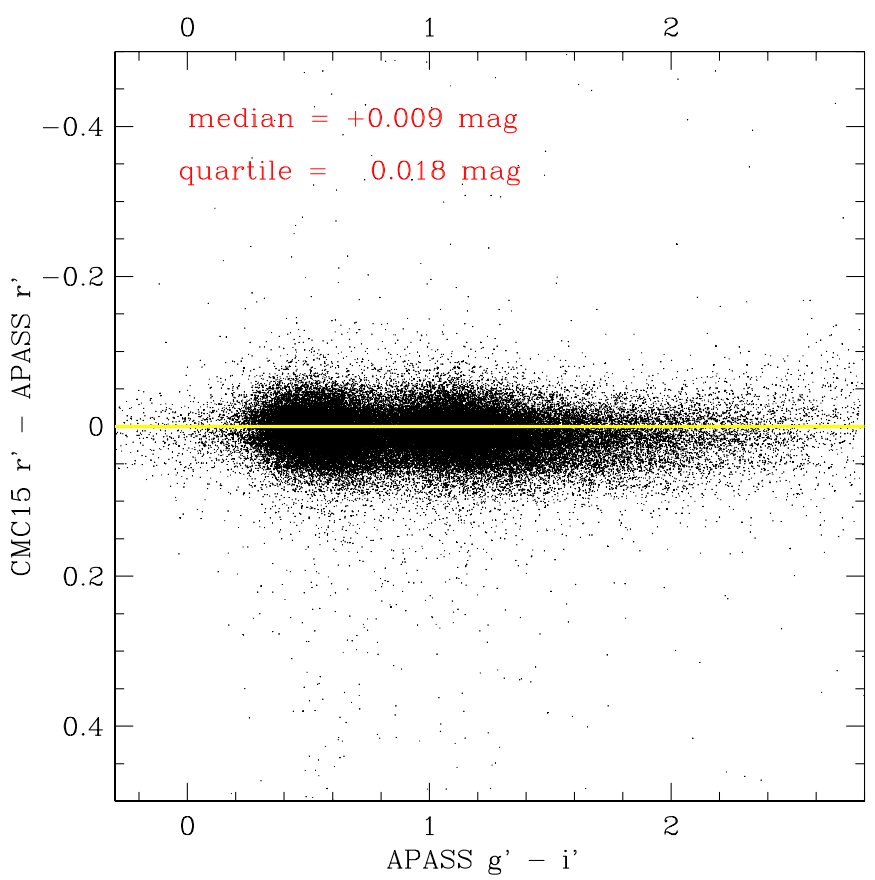

Figure 10. Comparison of the Carlsberg Meridian Catalog (CMC-15) and APASS $r^{\prime}$ magnitudes for the 262,972 RAVE stars common to both.

(A color version of this figure is available in the online journal.)

CMC-15 and APASS $r^{\prime}$ magnitudes strongly reinforces mutual confidence in them.

\subsection{Distribution in Magnitude and over the HR Diagram of RAVE Stars}

The distribution in APASS and 2MASS magnitudes for RAVE DR4 stars is shown in Figure 11, and it is characterized by the following median values (which are not affected by potential saturation problems at the very bright end of the distributions):

$$
\begin{aligned}
& \operatorname{med}(B)=12.654 \\
& \operatorname{med}\left(g^{\prime}\right)=12.126 \\
& \operatorname{med}(V)=11.676 \\
& \operatorname{med}\left(r^{\prime}\right)=11.362 \\
& \operatorname{med}\left(i^{\prime}\right)=11.033 \\
& \operatorname{med}(J)=9.802 \\
& \operatorname{med}(H)=9.330 \\
& \operatorname{med}(K)=9.214
\end{aligned}
$$

The stars for the RAVE input catalog were selected on the basis of their I-band magnitude (coming from DENIS, or SuperCOSMOS catalogs, or extrapolated from other sources like Tycho-2), generally within the boundaries $9 \leqslant I \leqslant 12$. To avoid problems from fiber cross-talk, the targets for any given RAVE observation should span the strictest possible range in magnitude that would still provide enough targets within the $6^{\circ}$ field of view to cover all available fibers. This led to grouping the targets in the RAVE input catalog over four distinct ranges in $I$-band magnitude (nominally 9.0-10.0, 10.0-10.75, 10.75-11.5, and 11.5-12.0). The corresponding tailing of the actual observations is apparent in the segmented distribution affecting the $i^{\prime}$ panel of Figure 13. The presence of segmentation in other bands declines with increasing wavelength separation from the $i^{\prime}$ band. It is noteworthy that the distribution in the $B$ 

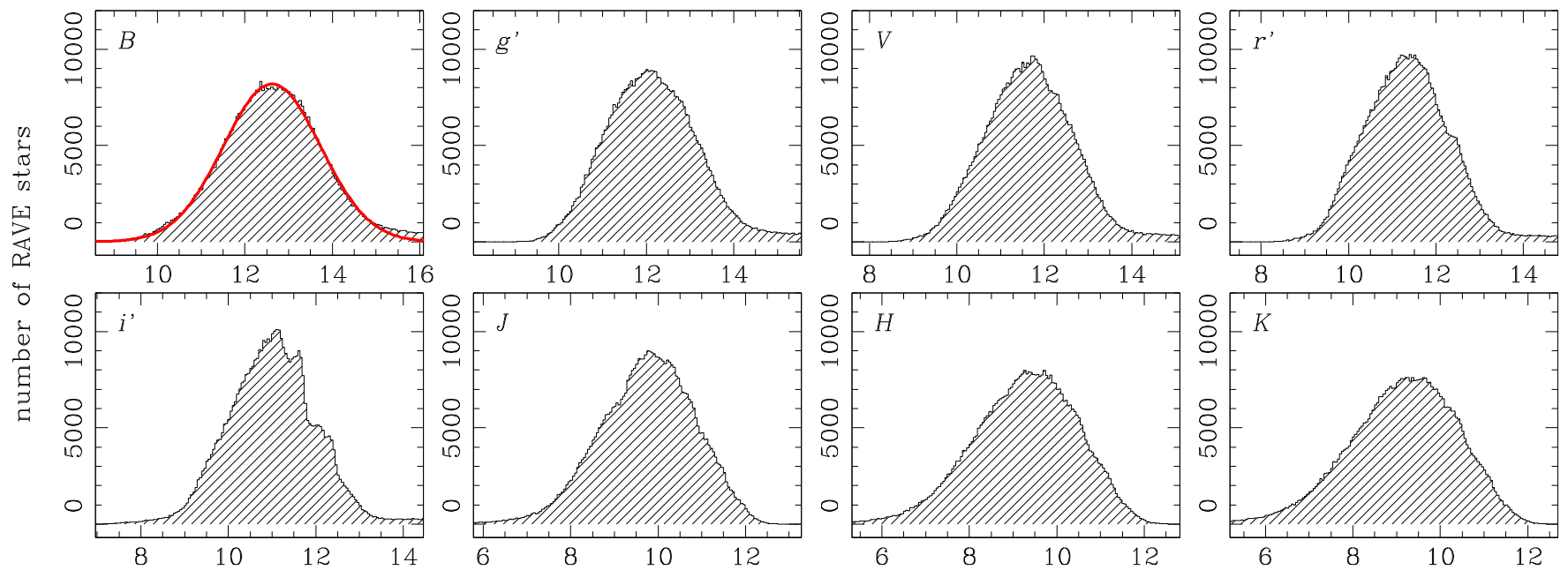

Figure 11. Distribution ( $\triangle \mathrm{mag}=0.05$ bins) in APASS $B V g^{\prime} r^{\prime} i^{\prime}$ and 2MASS $J H K$ magnitudes for RAVE DR4 stars. The segmented distribution in $i^{\prime}$ band is the result of RAVE stars being selected from different input catalogs and the observations being executed according to $I$-band brightness intervals. A Gaussian is overplotted for reference to the $B$-band distribution.

(A color version of this figure is available in the online journal.)
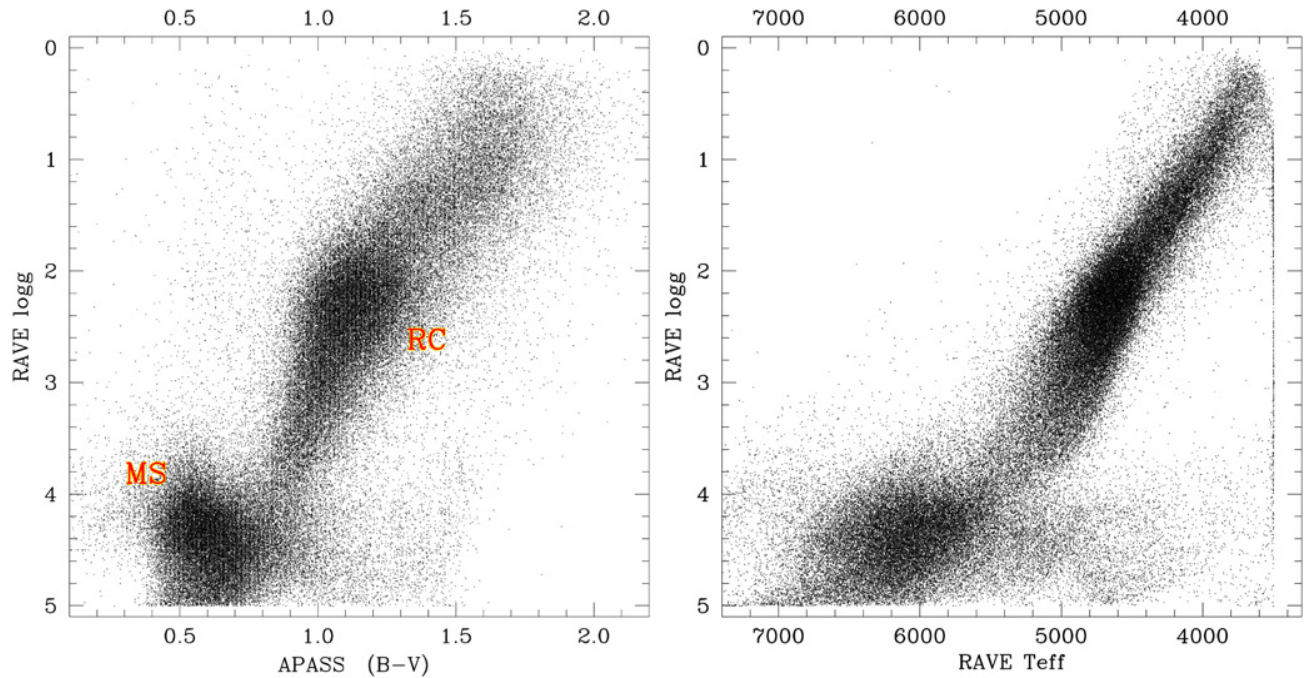

Figure 12. Distribution of RAVE stars on the spectroscopic $T_{\text {eff }}$ vs. $\log g$ plane (right) and distribution replacing $T_{\text {eff }}$ with APASS $B-V$ (left). The same stars are plotted on both panels.

(A color version of this figure is available in the online journal.)

band is closely matched by a Gaussian (overplotted) centered on $B=12.62$ and with $\sigma=1.11$ mag.

The distribution of RAVE stars over the HR diagram is shown in Figure 12, and is dominated by closer, hotter, and higher Galactic latitude main-sequence/turn-off (MS/TO) stars (the cloud of points centered at $B-V=0.6, \log g=4.4$ ), and by more distant, cooler, and lower Galactic latitude red giant branch and red clump stars (the cloud of points centered at $B-V=1.1, \log g=2.3$ ).

\section{FITTING TO A SYNTHETIC PHOTOMETRIC GRID}

To extract information about the observed stars, we performed a $\chi^{2}$ fit of APASS+2MASS photometry against a library of purposefully built synthetic photometry. The source stellar energy distributions are the atmospheric flux models of Castelli \& Kurucz (2003). Their model atmospheres are the basis of the Munari et al. (2005) synthetic spectral atlas, which has been used to derive atmospheric parameters from spectra in RAVE Data Releases 2 and 3. These models have been extensively used in the literature, including the recent work by Bessell \& Murphy (2012) on revised passbands and zero points for UBVRI, Hipparcos, and Tycho photometry.

The atmospheric flux models of Castelli \& Kurucz (2003) cover, at regular steps, a wide portion of the atmospheric parameter space along the effective temperature $\left(T_{\text {eff }}\right)$, surface gravity $(\log g)$, and metallicity $([\mathrm{M} / \mathrm{H}])$. Two values of the enhancement in $\alpha$ elements $([\alpha / \mathrm{M}])$ are considered $(0.0$ and +0.4$)$, while the microturbulence is constant at $2 \mathrm{~km} \mathrm{~s}^{-1}$. To increase the density of grid points in the range of temperatures covered by the vast majority of RAVE stars, the original atmospheric flux models for $T_{\text {eff }} \leqslant 9750 \mathrm{~K}$ have been parabolically interpolated to $\Delta T_{\text {eff }}=50 \mathrm{~K}, \Delta \log g=0.25 \mathrm{dex}, \Delta[\mathrm{M} / \mathrm{H}]=0.1$, and a third value $(+0.2)$ for $[\alpha / \mathrm{M}]$ has been derived from linear interpolation of source values $(0.0$ and +0.4$)$. For hotter temperatures $\left(T_{\text {eff }}>9750\right)$, where less than $1 \%$ of all RAVE stars are found, only one interpolated midpoint has been added between the original Castelli \& Kurucz (2003) grid points. The atmospheric flux models have then been reddened according to 64 different values of $E_{B-V}$, from 0.0 to 2.0, with a finer step at lower 
reddenings $\left(\Delta E_{B-V}=0.005\right)$ that progressively widens with increasing extinction (the last two points at $E_{B-V}=1.75$ and 2.00). Considering the high Galactic altitude of RAVE target stars, for the interstellar reddening law we adopted the standard $R_{V}=A_{V} / E_{B-V}=3.1$ law as formulated by Fitzpatrick (1999). A total of 13 million interpolated and reddened atmospheric flux models were used to build the photometric synthetic library.

The magnitudes in the eight bands of interest (Landolt $B, V$; Sloan $g^{\prime}, r^{\prime}, i^{\prime}$; and 2MASS $J, H, K$ ) were obtained for all input atmospheric flux models by direct integration:

$$
\operatorname{mag}=-2.5 \log \frac{\int 10^{\left(-0.4 R_{\lambda} E_{B-V}\right)} I(\lambda) S(\lambda) d \lambda}{\int S(\lambda) d \lambda}+\text { const }
$$

where $I(\lambda)$ is the flux distribution of the atmospheric model and $S(\lambda)$ is the transmission profile of the given photometric band. The zero points were set to match the observed values for the primary photometric standard of the Sloan system BD+17.4708, ${ }^{24}$ for which there are several published determinations of the atmospheric parameters. The corresponding mean values are $T_{\text {eff }}=5980 \mathrm{~K}, \log g=3.85$ and $[\mathrm{M} / \mathrm{H}]=-1.72$. An $[\alpha / \mathrm{Fe}]=$ +0.4 atmospheric flux model for these parameters was obtained by parabolic interpolation from Castelli \& Kurucz (2003), and it was reddened by $E_{B-V}=0.010$ following Ramírez et al. (2006). The zero points in Equation (2) then follow from the requirement that the computed values match the observed values $B=9.911, V=9.467, g^{\prime}=9.640, r^{\prime}=9.350, i^{\prime}=9.250$ (from Fukugita et al. 1996; Smith et al. 2002), and $J=8.435$, $H=8.108, K=8.075$ (2MASS catalog) for the BD+17.4708 standard.

To derive the best matching atmospheric parameters and reddening, the observed APASS + 2MASS $B, V, g^{\prime}, r^{\prime}, i^{\prime}, J, H, K$ magnitudes (mag*) were $\chi^{2}$ fitted to the synthetic photometric grid:

$$
\chi^{2}=\sum_{i} \frac{\left(\operatorname{mag}_{i}^{*}-\operatorname{mag}_{i}\right)^{2}}{\epsilon_{i}^{2}}
$$

where $i$ sums over the eight photometric bands and $\epsilon_{i}$ are the individual errors quoted in APASS and 2MASS catalogs. Extensive simulations on synthetic data and analysis of real data on RAVE stars shows that the great density of points in the matched synthetic photometric library produces $\chi^{2}$ minima which are symmetric around their core. To derive the best fit values, we therefore averaged over the five deepest points within the $\chi^{2}$ minimum.

\section{TEMPERATURE-REDDENING DEGENERACY}

The observed spectral energy distribution of a normal star is primarily dominated by the combined effect of temperature and reddening. The two cannot be fully disentangled from optical+IR photometric data alone. This is demonstrated in Figure 13, where the results of a Monte Carlo simulation are presented. We took the photometry of one atmospheric flux model (from the 13,244,000 entries of the synthetic photometric reference grid described in the previous section), Monte Carlo perturbed it according to a Gaussian distribution of errors (with $\sigma$ given by the values in Equation (1)), and $\chi^{2}$ fitted it back to the photometric reference grid. Figure 13 presents the distribution of the fitting results on three separate $T_{\mathrm{eff}}-E_{B-V}$ planes to highlight

\footnotetext{
24 Bohlin \& Gilliland (2004b) provided an accurate absolute flux distribution of this star based on Hubble Space Telescope data.
}

the effect of alpha-enhancement, surface gravity, and metallicity, while the last panel on each row shows the surface gravity versus metallicity correlation.

In the Monte Carlo simulations of Figure 13, we considered two cases, a main-sequence/turn-off star $\left(T_{\text {eff }}=5800 \mathrm{~K}\right.$, $\log g=4.0,[\alpha / \mathrm{Fe}]=0)$ and a red clump $\operatorname{star}\left(T_{\mathrm{eff}}=4650 \mathrm{~K}\right.$, $\log g=2.75,[\alpha / \mathrm{Fe}]=0)$, either metal-rich $([\mathrm{M} / \mathrm{H}]=+0.2)$ or metal-poor $([\mathrm{M} / \mathrm{H}]=-0.6)$, and suffering from $E_{B-V}=0.06$ or $E_{B-V}=0.34$ reddening, for a total of eight different combinations. The results of Figure 13 show that broadband photometry, even if widely distributed in wavelength over the optical and infrared ranges and of state-of-the-art accuracy, cannot accurately disentangle temperature from reddening-the two are intimately degenerate - and that the possibility of constraining gravity and metallicity is, at best, marginal. This is not surprising since we have at least five variables $\left(E_{B-V}, T_{\text {eff }}\right.$, $\log g,[\mathrm{M} / \mathrm{H}]$, and $[\alpha / \mathrm{Fe}])$ and only eight data points with which to constrain them (whereas spectra can count on hundreds of absorption lines as input). Here we considered the ideal case of $\chi^{2}$ fitted synthetic versus synthetic data. In the real world, with real stars, there are several additional variable factors to further confuse the picture: (1) a fraction of the real stars are binaries, merging the light of two or more stars of different properties (including the possibility of different circumstellar reddening, e.g., Allen 1983); (2) the cosmic dispersion of real stars (e.g., in chemical partition or micro-turbulence) cannot be accounted for by any synthetic photometric grid; (3) peculiarities like chromospheric activity (a common feature of young main-sequence stars), winds, and circumstellar material (frequent in cool giants, yellow supergiants, or hotter stars), or even the latitudinal dependence of colors on fast rotating stars (e.g., Golay 1974; Bohlin \& Gilliland 2004a); and the (4) differences in shapes that the wavelength dependence of interstellar reddening can take over the wavelength range of interest (from $R_{V}=2.1$ to $R_{V}=5.0$, e.g., Cardelli et al. 1989; Fitzpatrick 1999). These uncertainties are added to the known difficulties of synthetic spectra to accurately reproduce observed ones, especially at the borders of the explored parameter space.

In the next section, we will provide several different values of temperature and reddening for RAVE stars. In addition to those values derived by fitting APASS+2MASS data simultaneously for both temperature and reddening, we will also fix the reddening and derive the temperature and, conversely, fix the temperature and derive the reddening, so as to provide the reader with a range of options.

\section{DUST MODEL PARAMETERIZED ON STANDARD STARS}

To derive the temperature once the reddening is fixed, we introduce a simple reddening model. We assume that the low reddening affecting the high Galactic latitude RAVE stars originates from a homogeneous slab of dust, extending symmetrically for $d$ parsecs above and below the Galactic plane, with the Sun positioned on the Galactic plane. In this model, sketched in Figure 14, the amount of reddening is directly proportional to the length traversed by the line-of-sight within the dust slab (indicated by the thicker portions of the linesof-sight in Figure 14). The model is completely defined by the thickness of the slab $(d)$, the total reddening at the pole ( $E_{B-V}^{\text {poles }}$, the same value for the north and south poles), and the reddening law. We take the reddening law to be the standard $R_{V}=3.1$, valid for the diffuse interstellar medium (Savage \& 

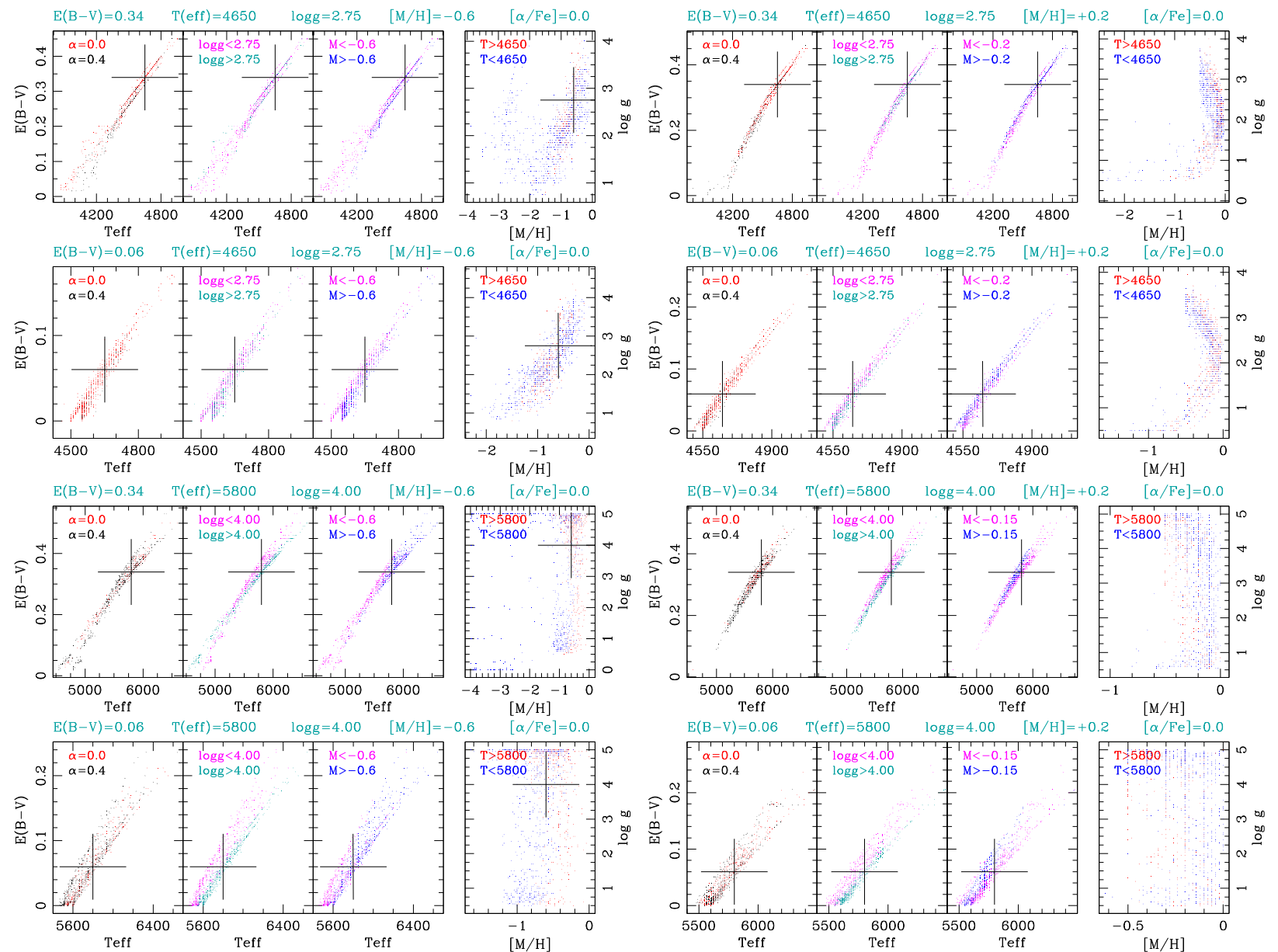

Figure 13. Degeneracy between reddening and temperature on multi-band photometry of stars. The $B V g^{\prime} r^{\prime} i^{\prime} J H K$ magnitudes of eight synthetic atmospheric flux distributions (for a red clump star and a main-sequence star, either metal-rich or metal-poor, and either significantly or marginally reddened) are Monte Carlo perturbed according to APASS and 2MASS typical errors for RAVE stars (cf Equation (1) and Figure 3), and then subjected to a $\chi^{2}$ fit to the synthetic photometric library (see Section 4 for details). The crosses mark the position of the reference unperturbed model.

(A color version of this figure is available in the online journal.)

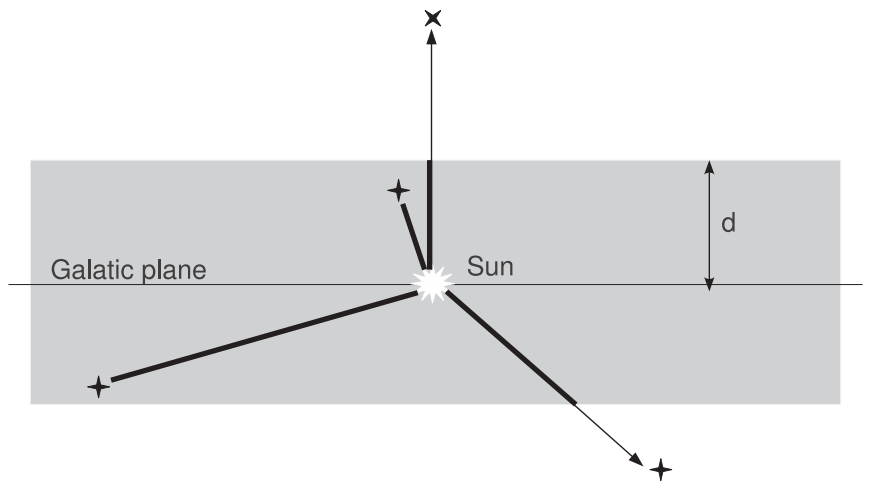

Figure 14. Outline of the simple Galactic dust distribution model described in Section 5 and used to statistically account for the interstellar extinction affecting high Galactic latitude RAVE stars.

Mathis 1979). Investigation of the actual 3D Galactic reddening map as inferred from APASS+2MASS data for RAVE stars will be carried out elsewhere in this series of papers. It is worth noting that typical RAVE stars are at high Galactic latitudes and at a significant distance from the Sun; thus most of them lie outside the dust slab. The distribution of dust within the slab is not important; we can characterize it with just two parameters: its thickness and its extinction at the poles. Also the presence of the local interstellar bubble around the Sun (e.g., Abt 2011 and references therein) is of little concern here. All RAVE and calibration stars below are considered to be well beyond it.

To derive the amount of total reddening at the poles and constrain the thickness of the dust slab, we considered two samples of stars, characterized by having both accurate atmospheric parameters (derived from high resolution, high $\mathrm{S} / \mathrm{N}$ spectroscopy) and accurate APASS and 2MASS photometry. A first sample comes from Ruchti et al. (2010, 2011a, 2011b), and a second sample is composed of stars from the PASTEL database Soubiran et al. (2010). Distances to these stars were obtained from isochrone fitting as described by Zwitter et al. (2010). These distances are homogeneous with those to RAVE stars computed in the same way (see below). The sample of Ruchti+PASTEL stars with unsaturated APASS+2MASS magnitudes is composed by 242 stars. They are distributed similarly to RAVE stars in terms of temperature and surface gravity: those hotter than $5300 \mathrm{~K}$ are generally main sequence objects, while giants dominates at lower temperatures. In both the RAVE survey and the Ruchti+PASTEL sample the number of giants is larger than the main sequence stars. They however differ significantly in metallicity: the median metallicity of Ruchti+PASTEL main sequence stars is -0.85 , and -1.41 is that of giants, those of RAVE stars are -0.17 and -0.43 , respectively. When unsaturated APASS photometry will be available for stars up to 
$7.5 \mathrm{mag}$, it will be possible to include a larger proportion of standard stars of higher metallicity.

The APASS+2MASS data for the stars in the Ruchti+PASTEL sample were subjected to the $\chi^{2}$ fitting described in Section 3. A dense grid of values for the thickness of the slab $(d)$ and the total reddening at the pole $\left(E_{B-V}^{\text {poles }}\right)$ was explored. The photometric temperature derived from the $\chi^{2}$ fitting was then compared to the spectroscopic values, and the $\Delta T_{\text {eff }}$ difference evaluated. The values of $d$ and $E_{B-V}^{\text {poles }}$ that best represent the Galactic extinction are those that tend to null the trend of $\Delta T_{\text {eff }}$ with $T_{\text {eff }}$, and that minimize both the median value and the rms of $\Delta T_{\text {eff }}$.

A value of $E_{B-V}^{\text {poles }}=0.036 \pm 0.003$ and $d=140 \pm 30 \mathrm{pc}$ are found. It is worth noting that the $\chi^{2}$ fitting assumes the reddening affecting the Sloan primary photometric standard BD+17.4708 to be $E_{B-V}=0.010$ following Ramírez et al. (2006). Any deviation from $E_{B-V}=0.010$ of the reddening affecting $\mathrm{BD}+17.4708$ should be added to $E_{B-V}^{\text {poles }}$. In the existing literature, various methods have been used to derive $E_{B-V}^{\text {poles }}$ (stellar counts and colors, galaxy counts and colors, interstellar polarization). A probably incomplete list of available literature provides the following determinations (in chronological order): $E_{B-V}^{\text {poles }}=0.023$ by Straižys \& Kavaliauskaite (1967), 0.029 by Bond et al. (1971), 0.018 by Philip (1973), 0.054 by Holmberg (1974), 0.03 by Appenzeller (1975), 0.048 by Heiles (1976), 0.057 by Knude (1977), 0.00 by Burstein \& Heiles (1978), 0.060 by Lynga (1979), 0.024 by Albrecht et al. (1980), 0.04 by Nicolet (1982), 0.048 by de Vaucouleurs \& Buta (1983), 0.00 by McFadzean et al. (1983), 0.050 by Eggen (1995), 0.055 by Berdyugin et al. (2001), and $>0.043$ by Berdyugin et al. (2004). Their mean value is $E_{B-V}^{\text {poles }}=0.036(\sigma=0.019$, and 0.005 as the error of the mean), the same value we found from APASS+2MASS data of Ruchti+PASTEL stars, which reinforces the confidence on the results of the present paper. The value of $140 \mathrm{pc}$ we found for $d$ well matches the scale height of the youngest stellar population of the Galaxy with which dust is intimately related.

Future extension of the APASS survey to cover stars up to 7.5 mag brightness will greatly increase the number of nearby spectroscopic standard stars, especially among the main sequence stars, and this will provide a stronger handle to fix $E_{B-V}^{\text {poles }}$ and $d$, as well as for investigating differences in the total extinction at the north and south Galactic poles.

\section{RESULTS}

The photometric temperature and reddening of RAVE stars have been computed according to the $\chi^{2}$ procedure described in Section 3 and the results are given in Table 1. The full table is available electronic only via CDS, the electronic edition of this journal and the RAVE Web site. The electronic version of the table will be kept updated in response to future RAVE and APASS data releases.

In addition to provide APASS astrometric positions and magnitudes (with their respective errors) for RAVE DR4 stars, Table 1 lists a series of values for the temperature and reddening computed under different conditions, outlined below. Table 1 lists results for 377,379 RAVE spectra (336,644 stars) and does not include the RAVE stars that have been classified as peculiar by Matijevič et al. (2012), which will be studied separately and in conjunction with epoch APASS photometry. For uniformity reasons, Table 1 also does not include the few RAVE stars for which data are missing in one or more of the
$B V g^{\prime} r^{\prime} i^{\prime} J H K$ photometric bands. By slab reddening in Table 1 , we mean the reddening produced by the line of sight to the given star traversing an homogeneous slab of interstellar dust, extending by $d=140 \mathrm{pc}$ on either sides of the Galactic plane (with the Sun centered on it), following the standard $R_{V}=3.1$ law and causing a total $E_{B-V}^{\text {poles }}=0.036 \mathrm{mag}$ at both Galactic poles. It is completely defined by the Galactic latitude and the distance to the given star. Finally, RAVE DR4 computed the atmospheric parameters $\left(T_{\text {eff }}, \log g\right.$ and $\left.[\mathrm{M} / \mathrm{H}]\right)$ from observed spectra following two different approaches, named kordo and sparv, which are described in Kordopatis et al. (2013) (where preference is given to kordo values) and that are both used in this paper.

The columns of Table 1 provide the following data. Column:

(1) RAVE name;

(2) APASS right ascension (equinox J2000, epoch 2013);

(3) its error (in $\operatorname{arcsec})$;

(4) APASS declination (equinox J2000, epoch 2013);

(5) its error (in arcsec);

(6) number $N$ of independent photometric nights during which the star was measured by APASS;

(7) $B$ mag;

(8) the $\sigma$ of the $N$ (Column 6) independent measurements;

(9) $V$ mag;

(10) the $\sigma$ of the $N$ (column 6) independent measurements;

(11) $g^{\prime} \mathrm{mag}$;

(12) the $\sigma$ of the $N$ (Column 6) independent measurements;

(13) $r^{\prime}$ mag;

(14) the $\sigma$ of the $N$ (Column 6) independent measurements;

(15) $i^{\prime}$ mag;

(16) the $\sigma$ of the $N$ (Column 6) independent measurements;

(17) $T_{\text {eff }}(\mathrm{K})$ computed by assuming the slab reddening corresponding to Binney et al. (2014) distance;

(18) its uncertainty $(K)$ expressed as the rms of the five deepest points within the $\chi^{2}$ minimum;

(19) $T_{\text {eff }}(\mathrm{K})$ computed by assuming the slab reddening corresponding to Zwitter et al. (2010) distance;

(20) its uncertainty $(K)$ expressed as the rms of the five deepest points within the $\chi^{2}$ minimum;

(21) $T_{\text {eff }}(\mathrm{K})$ computed by assuming the slab reddening corresponding to Zwitter et al. (2010) distance, and fixing the values of $\log g$ and $[\mathrm{M} / \mathrm{H}]$ to "kordo" values from RAVE DR4;

(22) its uncertainty $(K)$ expressed as the rms of the five deepest points within the $\chi^{2}$ minimum;

(23) $T_{\text {eff }}(\mathrm{K})$ computed by assuming the slab reddening corresponding to Zwitter et al. (2010) distance, and fixing the values of $\log g$ and $[\mathrm{M} / \mathrm{H}]$ to "sparv" values from RAVE DR4;

(24) its uncertainty $(K)$ expressed as the rms of the five deepest points within the $\chi^{2}$ minimum;

(25) $T_{\text {eff }}(\mathrm{K})$ computed by assuming null reddening;

(26) its uncertainty $(K)$ expressed as the rms of the five deepest points within the $\chi^{2}$ minimum;

(27) $T_{\text {eff }}(\mathrm{K})$ computed by letting the $\chi^{2}$ to simultaneously fit $E_{B-V}$

(28) its uncertainty $(K)$ expressed as the rms of the five deepest points within the $\chi^{2}$ minimum;

(29) slab $E_{B-V}$ reddening for Binney et al. (2014) distance;

(30) slab $E_{B-V}$ reddening for Zwitter et al. (2010) distance;

(31) $E_{B-V}$ (mag) computed by letting the $\chi^{2}$ to simultaneously fit $T_{\text {eff }}$; 
Table 1

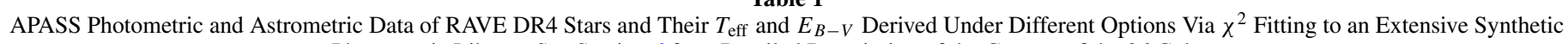
Photometric Library. See Section 6 for a Detailed Description of the Content of the 36 Columns

\begin{tabular}{|c|c|c|c|c|c|c|c|c|c|c|c|c|c|c|c|c|c|c|c|}
\hline \multicolumn{3}{|c|}{ (1) } & \multicolumn{2}{|c|}{ (2) } & (3) & \multicolumn{2}{|c|}{ (4) } & (5) & (6) & (7) & (8) & (9) & (10) & (11) & (12) & (13) & (14) & (15) & (16) \\
\hline \multicolumn{3}{|c|}{ RAVE } & \multicolumn{2}{|c|}{ RA } & err & \multicolumn{2}{|c|}{ DEC } & err & $\mathrm{N}$ & B & & $\mathrm{V}$ & & $g^{\prime}$ & & $r^{\prime}$ & & $i^{\prime}$ & \\
\hline \multicolumn{3}{|c|}{ J041407.8-282210 } & \multicolumn{2}{|c|}{63.532745} & 0.134 & \multicolumn{2}{|c|}{28.369382} & 0.088 & 4 & 12.957 & 0.019 & 12.348 & 0.005 & 12.599 & 0.017 & 12.209 & 0.014 & 12.100 & 0.009 \\
\hline (17) & (18) & (19) & (20) & (21) & (22) & (23) & (24) & (25) & (26) & (27) & (28) & (29) & (30) & $(31)$ & (32) & (33) & (34) & (35) & (36) \\
\hline$T_{\mathrm{eff}}^{\text {slab, } B+}$ & & $T_{\text {eff }}^{\text {slab, } Z+}$ & & $T_{\mathrm{eff}}^{k}$ & & $T_{\mathrm{eff}}^{s}$ & & $T_{\text {eff }}^{\text {null }}$ & & $T_{\mathrm{eff}}^{\mathrm{fit}}$ & & $E_{B-V}^{\text {slab, } B+}$ & $E_{B-V}^{\text {slab, } Z+}$ & $E_{B-V}^{\mathrm{fit}}$ & & $E_{B-V}^{\text {kordo }}$ & & $E_{B-V}^{\text {sparv }}$ & \\
\hline 5910 & 22 & 5910 & 22 & 6190 & 42 & 6190 & 42 & 5730 & 27 & 5830 & 57 & 0.050 & 0.050 & 0.033 & 0.018 & 0.041 & 0.004 & 0.041 & 0.004 \\
\hline
\end{tabular}

(This table is available in its entirety in machine-readable and Virtual Observatory (VO) forms in the online journal. A portion is shown here for guidance regarding its form and content.)

(32) its uncertainty (mag) expressed as the rms of the five deepest points within the $\chi^{2}$ minimum;

(33) $E_{B-V}$ (mag) computed by fixing $T_{\text {eff }}, \log g$ and $[\mathrm{M} / \mathrm{H}]$ to "kordo" values from RAVE DR4;

(34) its uncertainty (mag) expressed as the rms of the five deepest points within the $\chi^{2}$ minimum;

(35) $E_{B-V}$ (mag) computed by fixing $T_{\text {eff }}, \log g$ and $[\mathrm{M} / \mathrm{H}]$ to "sparv" values from RAVE DR4;

(36) its uncertainty (mag) expressed as the rms of $E_{B-V}$ of the five deepest points within the $\chi^{2}$ minimum.

Some remarks to Table 1 . The uncertainties of APASS photometry reported in Columns $8,10,12,14$, and 16 are the $\sigma$ of the $N$ independent measurements in that band, so that the error $(\epsilon)$ on the reported magnitude should be computed as $\sigma / \sqrt{N}$. When a "-" sign precedes the value of $\sigma$, it means that less than $N$ observations have been obtained in that specific band (in performing the $\chi^{2}$ procedure above described, we have assumed $\epsilon_{i}=\sigma_{i}$ in such a case, equivalent to assume that only one observation was obtained in that band). When neither a valid Zwitter et al. (2010), nor a Binney et al. (2014) distance exists for a given RAVE star (as for the stars included in the first RAVE Data Release), its distance is set to 0 and consequently the corresponding slab $E_{B-V}$ reddening is null. The error in right ascension of APASS positions, expressed in arcseconds, already takes into account the declination projection factor.

When 0.0 is listed as the rms of the five deepest points within the $\chi^{2}$ minimum for either $T_{\text {eff }}$ or $E_{B-V}$, it means these points differ only in the other fitted parameters (e.g., $\log g,[\mathrm{M} / \mathrm{H}]$, $[\alpha / \mathrm{Fe}])$. Unless otherwise indicated (see above), in computing the columns of Table $1 \log g,[\mathrm{M} / \mathrm{H}]$, and $[\alpha / \mathrm{Fe}]$ are let free to be fitted. Given the low sensitivity of broadband photometry to parameters other than $T_{\text {eff }}$ and $E_{B-V}$, we refrain from listing the fitting results for $\log g,[\mathrm{M} / \mathrm{H}]$ and $[\alpha / \mathrm{Fe}]$. We plan to reconsider this point at a later time when (1) final APASS products will be released that will further reduce its already excellent photometric errors, (2) extension of APASS toward brighter saturation limits will enable to test the sensitivity to $\log g,[\mathrm{M} / \mathrm{H}]$ and $[\alpha / \mathrm{Fe}]$ on a much larger set of well studied standard stars than currently available (the vast majority of them are brighter than $10 \mathrm{mag}$ ).

\section{DISCUSSION}

The temperature of RAVE stars obtained from $B V g^{\prime} r^{\prime} i^{\prime} J H K$ photometry is compared in Figure 15 to spectroscopic temperature from RAVE DR4. Photometric and spectroscopic temperatures agree well.

In the top four rows of Figure 15, this comparison is carried out separately for stars above and below $25^{\circ}$ in galactic latitude, which was the original limit of the RAVE survey to avoid the regions closer to the galactic plane where the reddening becomes relevant and increasingly patchy. The comparison is carried out separately for Zwitter et al. (2010) and Binney et al. (2014) distances to RAVE stars, and for the two different types of spectroscopic temperatures $T_{\mathrm{eff}}^{\text {sparv }}$ and $T_{\mathrm{eff}}^{\mathrm{kordo}}$ derived in RAVE DR4.

To filter out outliers and lower quality data, the comparison between photometric and spectroscopic temperatures in Figure 15 is carried out only for stars with best RAVE and APASS data. Only stars with 4 or more independent APASS photometric observations and fainter than the 10 mag safe limit for saturation risk are considered. These stars are further filtered out so that their RAVE DR4 spectra (1) have $\mathrm{S} / \mathrm{N} \geqslant 45$, (2) provided valid atmospheric parameters $\left(T_{\text {eff }}, \log g,[\mathrm{M} / \mathrm{H}]\right)$ via both sparv and kordo pipelines, (3) passed the chemical analysis pipeline of Boeche et al. (2011), and (4) do not show peculiarities according to Matijevič et al. (2012) pipeline. About 51,000 stars are plotted in Figure 15.

For galactic latitudes $|b| \geqslant 25^{\circ}$, Figure 15 shows that there is a negligible bulk difference between photometric temperatures (assuming the slab reddening described above) and spectroscopic $T_{\text {eff }}^{\text {sparv }}$ temperatures for both red giant/red clump (RC) and main-sequence/turn-off (MS/TO) stars, with no appreciable difference between Zwitter et al. (2010) and Binney et al. (2014) distances. Most of the RAVE stars are out of the dust slab, so their accurate distance does not matter here. The rms of the differences between the photometric and spectroscopic temperatures $(\Delta T)$ is $118 \mathrm{~K}$ for $\mathrm{RC}$ stars and $160 \mathrm{~K}$ for MS/ TO stars, both of which are comparable to the rms of the spectroscopic temperatures derived independently from multiple RAVE spectra of the same star. The comparison between photometric and $T_{\text {eff }}^{\text {kordo }}$ spectroscopic temperatures shows a minimal bulk difference for RC stars and a substantial one for MS/TO stars, while in both cases, the rms is similar to that found for $T_{\mathrm{eff}}^{\text {sparv }}$. The effect of fixing $\log g$ and $[\mathrm{M} / \mathrm{H}]$ to the spectroscopic values are explored in the fifth row of Figure 15 (panels $e, e^{\prime}$ ). While somewhat reducing the rms for both $\mathrm{RC}$ and MS/TO stars, it introduces an offset of about $40 \mathrm{~K}$ for $\mathrm{RC}$ stars.

At lower Galactic latitudes $\left(|b|<25^{\circ}\right)$, the simple slab reddening shows its limitation on RC stars by introducing a bulk difference between photometric and spectroscopic temperatures for both $T_{\mathrm{eff}}^{\text {sparv }}$ and $T_{\mathrm{eff}}^{\mathrm{kordo}}$ in addition to expanding the rms. At these low Galactic latitudes, compensation for reddening requires a more sophisticated approach, a true $3 \mathrm{D}$ model of the reddening distribution that will be derived elsewhere in this series.

The effect of assuming null reddening for RAVE stars is explored in row $f, f^{\prime}$ of Figure 15. The net effect is to lower the temperature of both MS/TO and RC stars because the redder 


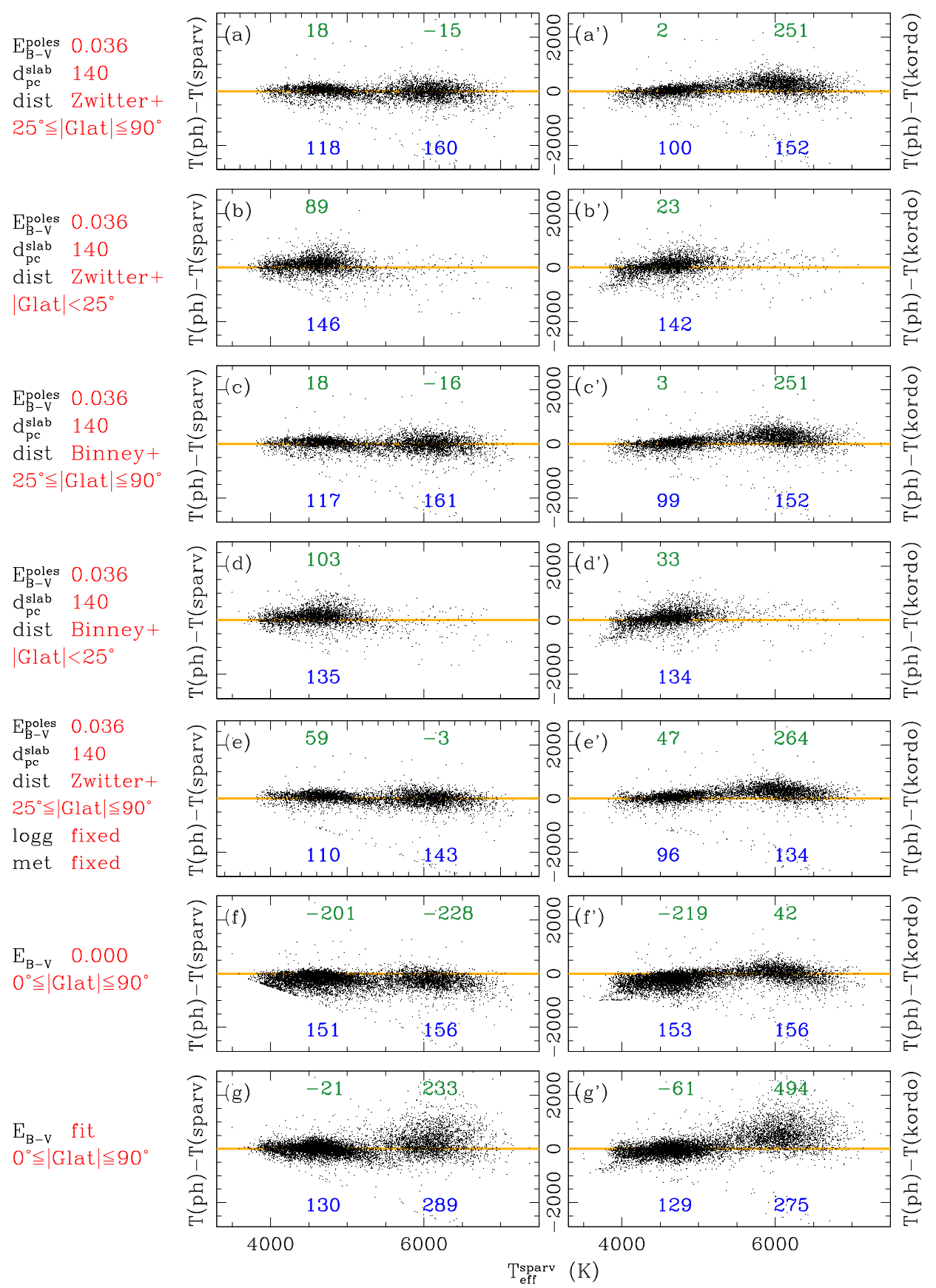

Figure 15. Difference between RAVE DR4 spectroscopic and APASS+2MASS photometric temperatures from Table 1 (cf. Section 7 for explanation and discussion). In each panel, the upper two numbers (in green) are the median value of the ordinate and the lower two (in blue) are the quartile values. They are listed separately for stars hotter and cooler than $5250 \mathrm{~K}$, a handy value dividing the RBG+RC stars (on the cooler side) from the MS/TO stars (on the hotter side).

(A color version of this figure is available in the online journal.)

energy distribution caused by extinction requires cooler stellar models to be fitted.

The last row of Figure 15 (panels $g, g^{\prime}$ ), compares spectroscopic and photometric temperatures when all parameters are simultaneously fitted by the photometric data and no assumption about the reddening is made. The comparison is good for RC stars, with no significant offset (at least against $T_{\text {eff }}^{\text {sparv }}$ ) and an rms similar to the cases when the reddening is fixed (first four rows of Figure 15). For MS/TO stars, the $T_{\text {eff }}$ so derived is instead unreliable, being characterized by a large offset and rms. The different behavior between RC and MS/TO stars can be traced to the fact that the maximum of the energy distribution for RC stars falls well within the wavelength range covered by the APASS+2MASS photometric bands, while for hotter MS/ TO stars this maximum tends to move to wavelengths shorter than those covered by APASS+2MASS.

By adopting the atmospheric parameters from spectroscopy, the fit to $B V g^{\prime} r^{\prime} i^{\prime} J H K$ returns the reddening. For RAVE DR4 stars, this is explored in Figure 16.

At higher Galactic latitudes sparv RAVE DR4 atmospheric parameters return a reddening that is very much in line with the slab reddening, as illustrated in panel $b$ of Figure 16. No trend is present and the offset is essentially null. Also kordo RAVE DR4 atmospheric parameters return a null trend (panel (a) of Figure 16), with a smaller rms but an appreciable offset. Closer to the Galactic plane (panels $d$ and $e$ of Figure 16), 


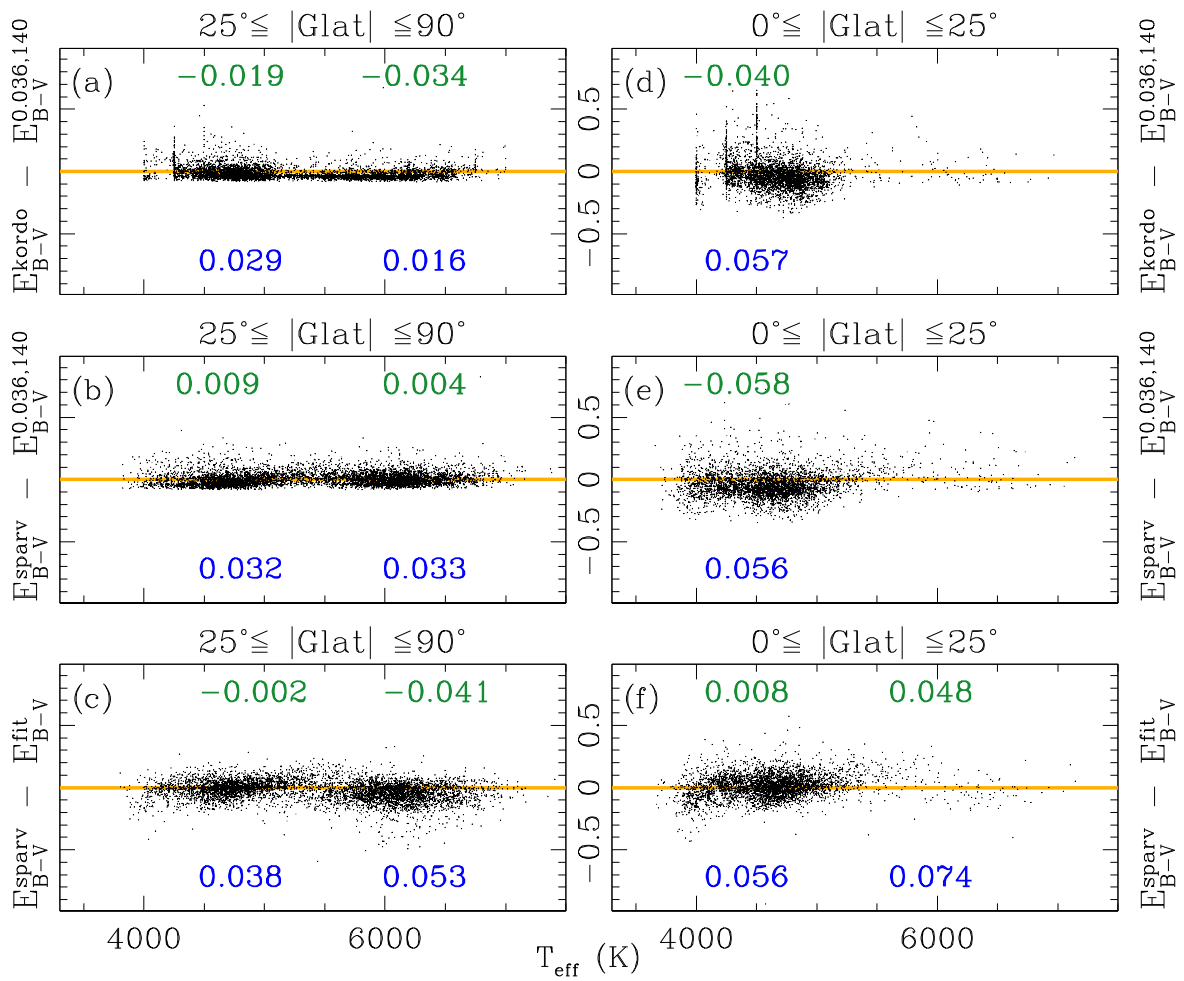

Figure 16. Upper two rows compare the slab reddening (cf. Section 5) to the reddening derived from $\chi^{2}$ fit to APASS+2MASS photometry fixing the atmospheric parameters to kordo and sparv values from RAVE DR4. The last row compares the reddening derived from simultaneous fitting to all parameters to that obtained by fixing the atmospheric parameters to sparv values from RAVE DR4. The panels at left focus on RAVE stars at $|b| \geqslant 25^{\circ}$, those at right to RAVE stars at $|b| \leqslant 25^{\circ}$.

(A color version of this figure is available in the online journal.)

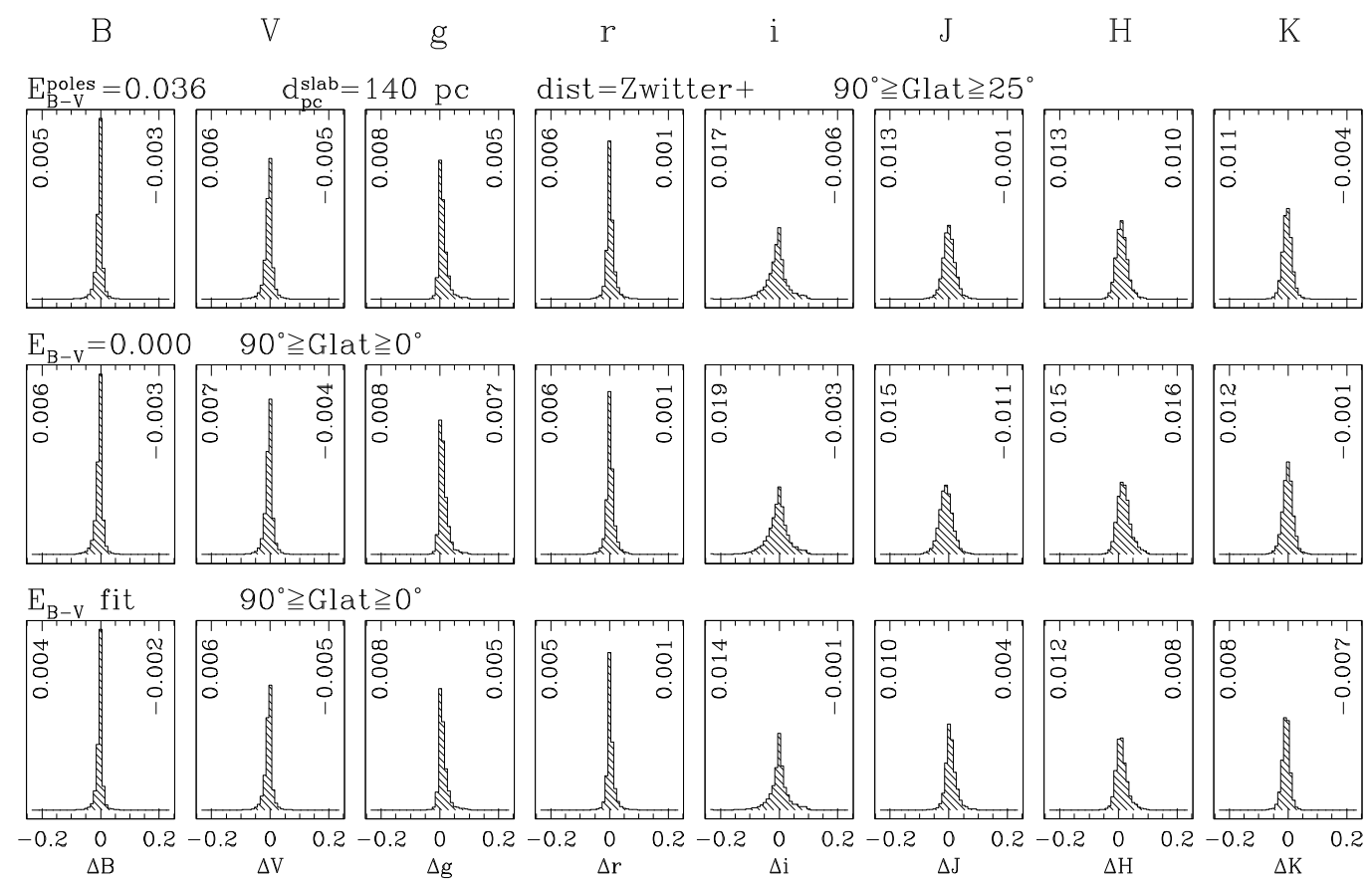

Figure 17. Distribution of the $\chi^{2}$ fitting residuals (mag) for, top to bottom, panels $a, f$, and $g$ of Figure 16. In each panel, the vertical number to the left is the rms (mag) and that to the right the median (mag).

comparisons with the slab reddening show the limitations of the $s l a b$ approach already noted. The last row of Figure 16 compares (separately for low and high Galactic latitudes) the reddening derived by fixing the atmospheric parameters to the sparv values and the reddening obtained when the atmospheric parameters are fitted simultaneously with $E_{B-V}$. As noted above for $T_{\text {eff }}$, the comparison is excellent for high Galactic latitude RC stars with a null trend and offset and only a marginal increase in rms. The comparison is less favorable for MS/TO stars and for low Galactic latitude RC stars.

Figure 17 displays the distribution of the differences between the observed magnitudes and those of the best fitting synthetic stellar model for three representative panels of Figure 15 (for other panels, they look similar). It is obvious that the 


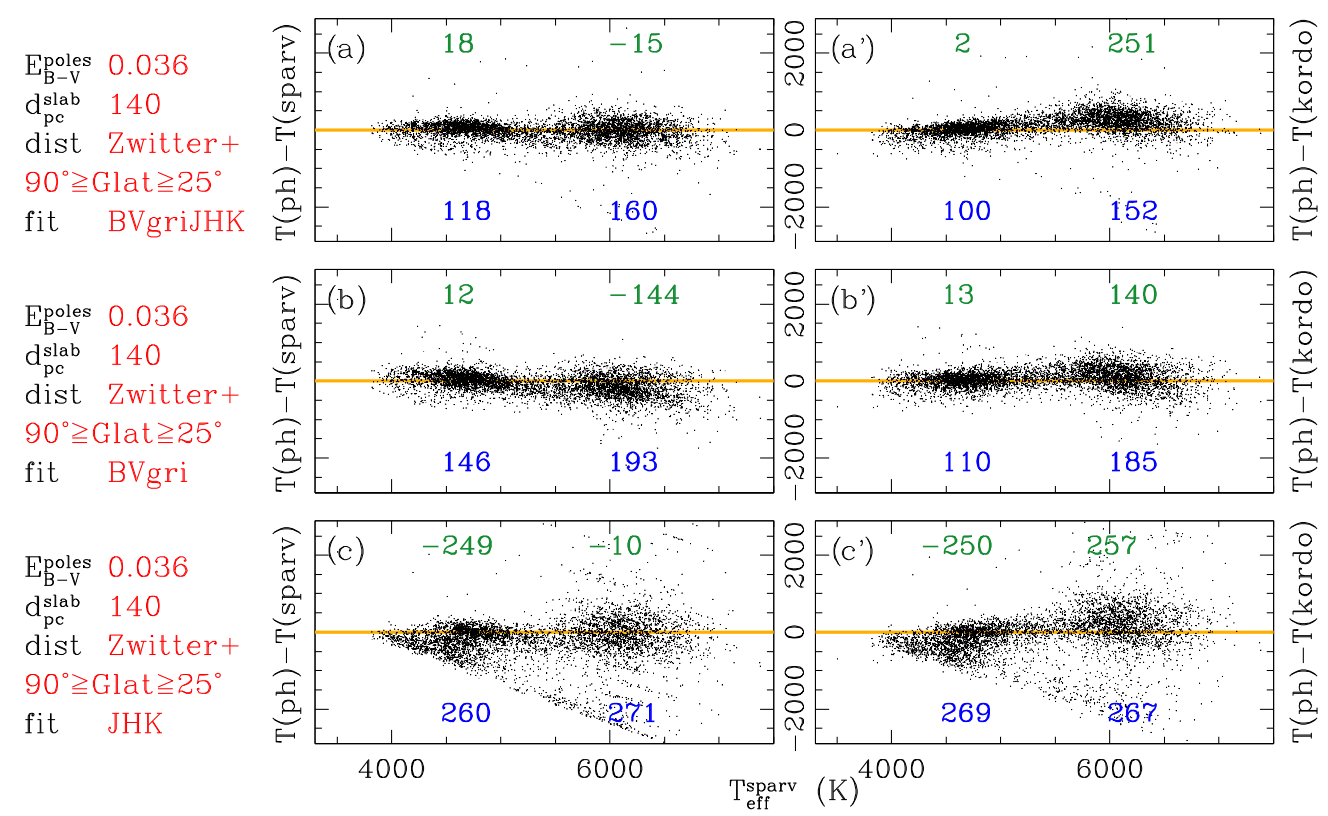

Figure 18. Effect of ignoring 2MASS (middle panels) or APASS data (bottom panels) in the $\chi^{2}$ fitting of RAVE DR4 stars. Panels (a), (a') are copied from Figure 16 for comparison purposes.

(A color version of this figure is available in the online journal.)

distributions for optical bands are much sharper than those for infrared bands. This reflects the fact that optical bands carry more information (closer to the peak of the energy distribution and where the effect of the reddening is larger) than infrared bands as well as the fact that the errors of 2MASS data are much larger than those for APASS (cf. Equation (1)).

The much greater importance of optical bands compared to infrared ones in constraining the temperature and reddening of stars is reaffirmed in Figure 18. Here the same computation performed for the first row of Figure 15 (its panels $(a)\left(a^{\prime}\right)$ are copied at the top of Figure 18 for comparison purposes), are repeated by $\chi^{2}$ fitting only the APASS optical data or only the 2MASS infrared data. It is quite obvious how in the case of optical-only data the overall picture is retained, while for IRonly data the fit is far less constrained.

\section{CONCLUSIONS}

The APASS all-sky, Landolt-Sloan $B V g^{\prime} r^{\prime} i^{\prime}$ photometric survey now provides the missing broadband optical photometric support to the RAVE spectroscopic survey and, coupled with 2MASS $J, H, K$ infrared data, it offers a wide wavelength coverage. The high astrometric and photometric accuracy of the APASS data has been verified on a large sample of standard and literature stars. With this first paper in the series, we provide $B V g^{\prime} r^{\prime} i^{\prime}$ data for the stars included in the latest RAVE DR4 data release, and derive temperature and reddening for them under different conditions by $\chi^{2}$ fitting to a densely populated synthetic photometric library. The comparison of photometric and spectroscopic temperatures of RAVE stars shows good agreement, indicating that photometric temperature can be either valid during or prior to spectroscopic analysis. RAVE stars at Galactic latitudes $|b| \geqslant 25^{\circ}$ display an $E_{B-V}$ reddening quite well approximated by a slab distribution of the interstellar dust that is characterized by $140 \mathrm{pc}$ extensions on either side of the Galactic plane and a reddening at the Galactic poles amounting to $E_{B-V}^{\text {poles }}=0.036$ mag. Future papers in this series will focus on RAVE peculiar stars, on the $3 \mathrm{D}$ structure of interstellar reddening as constrained by the APASS+2MASS photometry of RAVE stars, and on the results of the APASS extension to brighter magnitude limits and additional $\mathrm{u}^{\prime}, z^{\prime}$, and $Y$ photometric bands.

The referee, M. Bessell, is acknowledged for a careful reading and relevant suggestions. Funding for APASS has been provided by the Robert Martin Ayers Sciences Fund. We also particularly acknowledge support from the many volunteers and vendors. The APASS Web site is at http://www.aavso.org/apass. Funding for RAVE has been provided by the Australian Astronomical Observatory; the Leibniz-Institut fuer Astrophysik Potsdam (AIP); the Australian National University; the Australian Research Council; the French National Research Agency; the German Research Foundation (SPP 1177 and SFB 881); the European Research Council (ERC-StG 240271 Galactica); the Istituto Nazionale di Astrofisica at Padova; Johns Hopkins University; the National Science Foundation of the US (AST-0908326); the W. M. Keck Foundation; Macquarie University; the Netherlands Research School for Astronomy; the Natural Sciences and Engineering Research Council of Canada; the Slovenian Research Agency; the Swiss National Science Foundation; the Science \& Technology Facilities Council of the UK; Opticon; Strasbourg Observatory; and the Universities of Groningen, Heidelberg, and Sydney. The RAVE website is at http://www.rave-survey.org.

\section{REFERENCES}

Abt, H. A. 2011, AJ, 141, 165

Albrecht, R., \& Maitzen, H. M. 1980, A\&AS, 42, 9

Allen, D. A. 1983, MNRAS, 204, 113

Appenzeller, I. 1975, A\&A, 38, 313

Berdyugin, A., Piirola, V., \& Teerikorpi, P. 2004, A\&A, 424, 873

Berdyugin, A., \& Teerikorpi, P. 2001, A\&A, 368, 635

Bessell, M. S. 1990, PASP, 102, 1181

Bessell, M. S., \& Murphy, S. 2012, PASP, 124, 140

Binney, J., Burnett, B., Kordopatis, G., et al. 2014, MNRAS, 437, 351

Boeche, C., Siebert, A., Williams, M., et al. 2011, AJ, 142, 193 
Bohlin, R. C., \& Gilliland, R. L. 2004a, AJ, 127, 3508

Bohlin, R. C., \& Gilliland, R. L. 2004b, AJ, 128, 3053

Bond, H. E., Perry, C. L., \& Bidelman, W. P. 1971, PASP, 83, 643

Burstein, D., \& Heiles, C. 1978, ApJ, 225, 40

Cardelli, J. A., Clayton, G. C., \& Mathis, J. S. 1989, ApJ, 345, 245

Castelli, F., \& Kurucz, R. L. 2003, in IAU Symp. 210, Modelling of Stellar Atmospheres, ed. N. Piskunov, W. W. Weiss, \& D. F. Gray (San Francisco, CA: ASP), A20

de Vaucouleurs, G., \& Buta, R. 1983, AJ, 88, 939

Eggen, O. J. 1995, AJ, 109, 2229

Fiorucci, M., \& Munari, U. 2003, A\&A, 401, 781

Fitzpatrick, E. L. 1999, PASP, 111, 63

Fukugita, M., Ichikawa, T., Gunn, J. E., et al. 1996, AJ, 111, 1748

Golay, M. 1974, in Introduction to Astronomical Photometry (Dordrecht: Reidel)

Henden, A., \& Munari, U. 2000, A\&AS, 143, 343

Henden, A., \& Munari, U. 2001, A\&A, 372, 145

Henden, A., \& Munari, U. 2006, A\&A, 458, 339

Henden, A., \& Munari, U. 2014, Contrib. Astron. Obs. Skalnate Pleso, 43, 518

Henden, A., et al. 2014, AJ, submitted

Hillenbrand, L. A., Foster, J. B., Persson, S. E., \& Matthews, K. 2002, PASP, 114,708

Heiles, C. 1976, ApJ, 204, 379

Holmberg, E. B. 1974, A\&A, 35, 121

Knude, J. K. 1977, ApL, 18, 115

Kordopatis, G., Gilmore, G., Steinmetz, M., et al. 2013, AJ, 146, 134

Landolt, A. U. 2009, AJ, 137, 4186

Lynga, G. 1979, IAUS, 84, 87

Matijevič, G., Zwitter, T., Bienaymé, O., et al. 2012, ApJS, 200, 14
McFadzean, A. D., Hilditch, R. W., \& Hill, G. 1983, MNRAS, 205, 525

Mermilliod, J.-C., \& Mermilliod, M. 1994, Catalogue of Mean UBV Data on Stars (New York: Springer)

Moro, D., \& Munari, U. 2000, A\&AS, 147, 361

Munari, U., Bacci, S., Baldinelli, L., et al. 2012, BaltA, 21, 13

Munari, U., Henden, A., \& Frigo, A. 2014, NewA, 27, 1

Munari, U., \& Moretti, S. 2012, BaltA, 21, 22

Munari, U., Sordo, R., Castelli, F., \& Zwitter, T. 2005, A\&A, 442, 1127

Nicolet, B. 1982, A\&AS, 47, 199

Philip, A. G. D. 1973, in IAU Symp. 52, Interstellar Dust and Related Topics, ed. J. M. Greenberg \& H. C. van de Hulst (Dordrecht: Reidel), 263

Ramírez, I., Allende Prieto, C., Redfield, S., \& Lambert, D. L. 2006, A\&A, 459,613

Ruchti, G. R., Fulbright, J. P., Wyse, R. F., et al. 2010, ApJL, 721, L92

Ruchti, G. R., Fulbright, J. P., Wyse, R. F., et al. 2011a, ApJ, 737, 9

Ruchti, G. R., Fulbright, J. P., Wyse, R. F., et al. 2011b, ApJ, 743, 107

Savage, B. D., \& Mathis, J. S. 1979, ARA\&A, 17, 73

Sharon, C., Hillenbrand, L., Fischer, W., \& Edwards, S. 2010, AJ, 139, 646

Siebert, A., Williams, M. E. K., Siviero, A., et al. 2011, AJ, 141, 187

Smith, J. A., Tucker, D. L., Kent, S., et al. 2002, AJ, 123, 2121

Soubiran, C., Le Campion, J.-F., Cayrel de Strobel, G., \& Caillo, A. 2010, A\&A, 515, A111

Steinmetz, M., Zwitter, T., Siebert, A., et al. 2006, AJ, 132, 1645

Straižys, V. 1995, Multicolor Stellar Photometry (Tucson, AZ: Pachart)

Straižys, V., \& Kavaliauskaite, G. 1967, VilOB, 20, 3

Williams, M. E. K., Steinmetz, M., Sharma, S., et al. 2011, ApJ, 728, 102

Wylie de Boer, E., Freeman, K., Williams, M., et al. 2012, ApJ, 755, 35

Zwitter, T., Matijevič, G., Breddels, M. A., et al. 2010, A\&A, 522, A54

Zwitter, T., Siebert, A., Munari, U., et al. 2008, AJ, 136, 421 